\title{
A REVISION OF THE LOWER PENNSYLVANIAN Alethopteris lonchitica (auctorum) AND ITS IDENTITY WITH Alethopteris urophylla
}

\author{
Robert H. WAGNER and Carmen ÁLVAREZ- \\ VÁZQUEZ
}

Centro Paleobotánico, IMGEMA-Jardín Botánico de Córdoba, Avda. de Linneo, s/n, 14004 Córdoba (Spain); e-mail: cr1wagro@uco.es

\begin{abstract}
Wagner, R. H. \& Álvarez-Vázquez, C. 2008. A revision of the lower Pennsylvanian Alethopteris lonchitica (auctorum) and its identity with Alethopteris urophylla. [Revisión de la especie del Pensilvánico inferior Alethopteris lonchitica (auctorum) y su identificación con Alethopteris urophylla.] Revista Española de Paleontología, 23 (2), 157-192. ISSN 0213-6937.
\end{abstract}

\begin{abstract}
One of the most common lower Pennsylvanian (upper Namurian, lower Westphalian) pteridosperms is a species commonly recorded as Alethopteris lonchitica Schlotheim, 1820 ex Sternberg, 1825. However, the photographic illustration of the lectotype of Alethopteris lonchitica by Zodrow \& Cleal (1998) has allowed a clear distinction from Alethopteris lonchitica (auctorum) which corresponds, in the main, to Alethopteris urophylla (Brongniart, 1834) Göppert, 1836. This species is redescribed here, and its holotype is illustrated photographically for the first time. The published records, backed up by illustration, are analysed with reference to lists of synonymy. The geographic and stratigraphic distribution of Alethopteris urophylla is discussed, and illustrations of this species are provided from Nova Scotia, Scotland, England, Wales, the North of France, and NW as well as SW Spain. Alethopteris lonchitica was identified with Alethopteris lonchitifolia Bertrand, 1932 by Zodrow and Cleal, but the present writers prefer an identification with Alethopteris friedelii Bertrand, 1932. The corresponding taxonomic implications are discussed.
\end{abstract}

Keywords: Systematic palaeobotany, Alethopteris, lower Pennsylvanian, Canada, Scotland, England, Wales, France, Spain.

\section{RESUMEN}

Una de las especies de pteridosperma más citadas en el Pensilvánico inferior (Namuriense superior, Westfaliense inferior) es Alethopteris lonchitica Schlotheim ex Sternberg. La figuración fotográfica del lectotipo de esta especie en Zodrow \& Cleal (1998) ha permitido demostrar que la mayoría de los ejemplares atribuídos a Alethopteris lonchitica (auctorum) se identifican, en realidad, con Alethopteris urophylla (Brongniart, 1834) Göppert, 1836. En este trabajo se redescribe e ilustra fotográficamente, por primera vez, el holotipo de Alethopteris urophylla. Todos los registros avalados por ilustraciones se comentan en las listas de sinonimia. Se estudia también la distribución geográfica y estratigráfica de la especie, figurando ejemplares de Alethopteris urophylla procedentes de Nueva Escocia, Escocia, Inglaterra, País de Gales, Norte de Francia, y del NO y SO de España. Finalmente, se discute la identificación, hecha por Zodrow y Cleal, de Alethopteris lonchitica con Alethopteris lonchitifolia Bertrand, 1932; en este trabajo se defiende la sinonimia con Alethopteris friedelii Bertrand, 1932, y se analizan las implicaciones taxonómicas que esto supone.

Palabras clave: Taxonomía paleobotánica, Alethopteris, Pensilvánico inferior, Canadá, Escocia, Inglaterra, Gales, Francia, España.

\section{INTRODUCTION}

Alethopteris lonchitica, a species introduced by Schlotheim $(1804,1820)$ as Filicites lonchiticus, and referred to as Alethopteris lonchitidis by Sternberg (1825), has been commonly identified with an upper Namurian to lower Westphalian (lower Pennsylvanian) species of widespread occurrence in the Amerosinian Realm, i.e. the palaeoequatorial belt of Carboniferous times.

It has been apparent for some time that this was likely 
to be a misidentification since Schlotheim's specimen came from upper Westphalian strata in the Saar Coalfield in western Germany. Thomas \& Cleal (1993a: 115), quoting Wagner (1968: 107), opted for applying the name of Alethopteris urophylla to the upper Namurian-lower Westphalian taxon. They also suggested that the real Alethopteris lonchitica would be the same as Alethopteris lonchitifolia, Alethopteris ingbertensis Kessler, 1916, Alethopteris missouriensis White, 1899, Alethopteris densinervosa Wagner, 1968 and Alethopteris westphalensis Wagner, 1968. This identification is rejected in the present paper in favour of another species from Saarland, Alethopteris friedelii, a synonym of Alethopteris ambigua Lesquereux, 1879, according to Wagner (1968). Previously, Scheihing \& Pfefferkorn (1980) had placed in synonymy Alethopteris serlii (Brongniart, 1828a) Göppert, 1836, Alethopteris lonchitifolia, Alethopteris ingbertensis, Alethopteris missouriensis, Alethopteris densinervosa and Alethopteris westphalensis, referring all these species to the "Alethopteris serlii group". Most of these species had been grouped already by Wagner (1968: 172) as regional varieties of Alethopteris lonchitifolia. The total synonymy proposed by Scheihing \& Pfefferkorn (1980) was rejected by Zodrow \& Cleal (1998), who excluded Alethopteris serlii (in agreement with Wagner, 1968).

Zodrow \& Cleal (1998) published photographs of the type specimen of Alethopteris lonchitica which had been rediscovered in the collections of the Museum für Naturkunde in Berlin. In agreement with Thomas \& Cleal (1993a), they identified this species with Alethopteris lonchitifolia, which had also been described from Saarland. However, this identification is debatable, since Alethopteris lonchitifolia possesses more biconvex pinnules, with a denser nervation. Schlotheim's shows more parallel-sided, generally narrower pinnules, with a wider venation. This conforms better to the characters of Alethopteris friedelii, another taxon described from Saarland. The latter was regarded as a synonym of Alethopteris ambigua by Wagner (1968), who figured a wide range of specimens of this species from different parts of the palaeoequatorial belt (Amerosinian Realm) of Pennsylvanian times, from eastern North America to Asia Minor. This included a photographic illustration of the lectotype (figured originally as a drawing by Lesquereux, 1879: pl. XXXI, figs 1, 1a). Of course, identification with Alethopteris lonchitica implies that Alethopteris ambigua is a synonym.

The upper Namurian and lower Westphalian specimens which may be referred to as Alethopteris lonchitica (auctorum) (non von Schlotheim), are clearly different. Most of the specimens illustrated in the literature are comparable to Alethopteris urophylla, and the opportunity is taken here to refigure Brongniart's type specimen from Merthyr Tydfill in South Wales. Another species which is commonly regarded as identical is Alethopteris discrepans, from eastern Canada. New material from this area has recently become available, and is recorded in the present paper. The present writers also take the opportunity to figure comparable specimens from Scotland, England, the North of France, and two different parts of Spain. Good illustrations are already available from Britain (Crookall, 1955), northern France (Buisine, 1961) and western Germany (Josten, 1991).

\section{GENERAL COMMENTS ON Alethopteris lonchitica AND SIMILAR SPECIES}

The species Alethopteris lonchitica was introduced as Filicites lonchiticus by Schlotheim $(1804,1820)$, and illustrated by means of a composite drawing of several different pinna fragments occurring on two different faces of one and the same rock specimen from the upper Westphalian strata in Saar-Lorraine, a coal basin which straddles parts of Germany and France. The correct attribution of this species has always been problematical in view of the diagrammatic nature of Schlotheim's illustration, and palaeobotanists have usually followed Zeiller (1886: pl. XXXI, figs 1,1A) who identified it with a magnificent specimen from the lower Westphalian of the North of France. Additional, well preserved specimens from the North of France were published by Corsin (1932) and Buisine (1961), who monographed the alethopterids from this area. Buisine's exhaustive documentation of Alethopteris lonchitica apparently refers to the species figured and described by Zeiller (1886-88). There is a strong resemblance to Alethopteris urophylla, a species described from the lower Westphalian of South Wales, and which is commonly attributed to Alethopteris lonchitica in the literature. Records in the literature are most commonly under the name of Alethopteris lonchitica, even though this has been regarded as a misidentification (Wagner, 1968), a point of view which the photographic illustration of Schlotheim's type specimen has confirmed (Zodrow \& Cleal, 1998). A complex synonymy has been generated. This is analysed later in the present paper.

Earlier authors have generally ignored the type of Alethopteris lonchitica, which was reputedly lost, and Wagner (1968: 107) went so far as to suggest that Alethopteris lonchitica might have to be abandoned since its characters were difficult to establish from Schlotheim's diagrammatic drawing. In fact, the many records of specimens identified as Alethopteris lonchitica and the comprehensive lists of synonymy in Crookall (1955) and Buisine (1961) suggest that this species name was used for a variety of forms. Even so, Zeiller's (1886-88) interpretation of Alethopteris lonchitica normally prevailed. Its delimitation from other species, e.g. Alethopteris decurrens (Artis, 1825) Frech, 1880, has sometimes given rise to doubt. Gothan (1953) illustrated a wide range of specimens from the Ruhr District under the name of Alethopteris lonchitica, including 


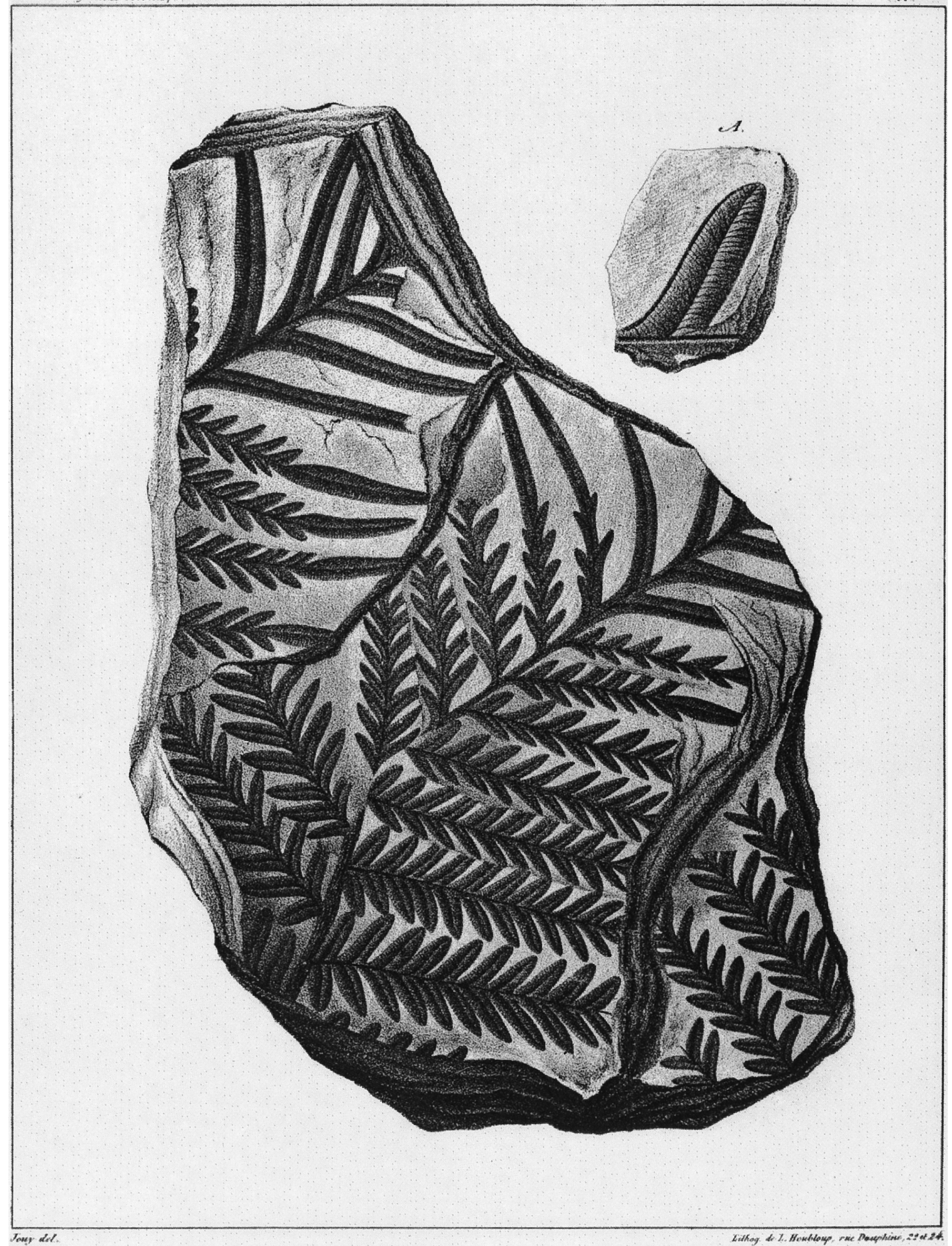

Figure 1. Copy of original plate of Pecopteris urophylla published by Brongniart (1834: pl. 86). 
an Alethopteris lonchitica forma serlii. This is perhaps one of the more extreme cases of a very broad interpretation of Alethopteris lonchitica (auctorum).

The rediscovery of the type of Alethopteris lonchitica in the Schlotheim Collection in the Museum für Naturkunde in Berlin, and its photographic illustration by Zodrow \& Cleal (1998) has allowed this species to become better known. These authors rejected the majority of published records of Alethopteris lonchitica, and focused on the various forms described from the Saar-Lorraine Coalfield by

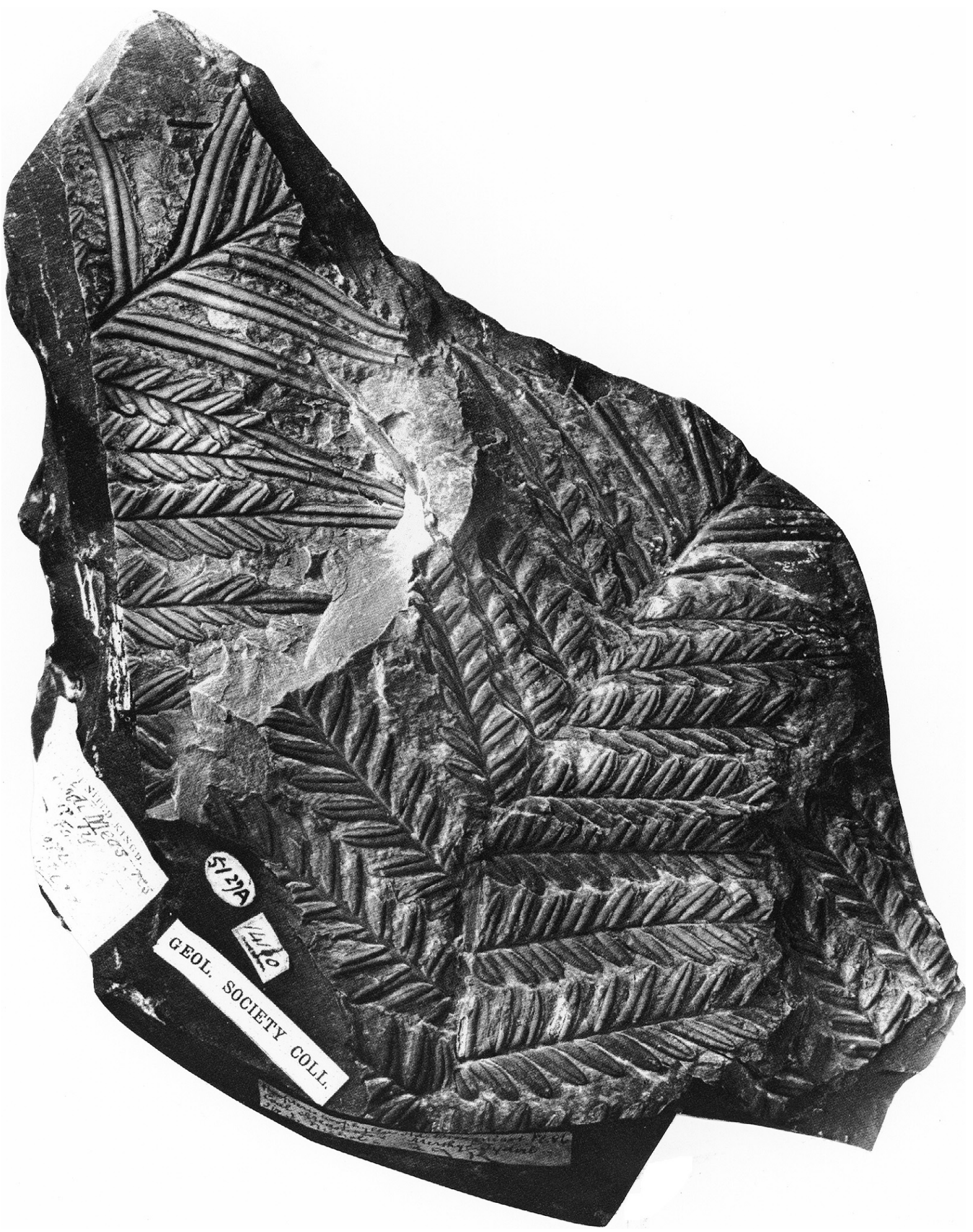

Figure 2. Photograph of Brongniart's type specimen of Pecopteris urophylla from Merthyr Tydfill in South Wales, lower Westphalian (x 1). Repository: Palaeontology Unit, British Geological Survey, Keyworth. Photograph: Barry Pigott, Sheffield University. 
Bertrand (1932). This is reasonable since Schlotheim's type specimen reputedly came from Saarland. They concluded that Alethopteris lonchitica would coincide with Alethopteris lonchitifolia. However, this seems an unfortunate choice, because there is a closer resemblance with Alethopteris friedelii, a species which has been placed in synonymy with Alethopteris ambigua by Wagner (1968). Pinnule shape, vein density, the presence of a compression border which relates to the thickness of pinnule lamina, all these characters shown by the type specimen of Alethopteris lonchitica coincide with those of Alethopteris ambigua. Unfortunately, Zodrow \& Cleal (1998) changed the meaning of Alethopteris ambigua so as to include specimens from the Sydney Basin in Nova Scotia, which are more properly regarded as belonging to Alethopteris lesquereuxii Wagner, 1968. Indeed, Zodrow \& Cleal (1998: 97) stated that where they used the name Alethopteris lesquereuxii in their earlier, biostratigraphical studies, this should now read Alethopteris ambigua. They also suggested that both species should be regarded as identical (or partly identical according to their list of synonymy for Alethopteris ambigua). They further compared with Alethopteris leonensis Wagner, 1964, which they suggested might be a descendant of Alethopteris ambigua. It is recalled that Alethopteris leonensis was placed in synonymy with Alethopteris virginiana Fontaine \& White, 1880, by Gillespie \& Pfefferkorn (1986), an identification which may be at least partly correct. Unfortunately, the diagrammatic nature of Fontaine \& White's (1880) illustrations of Alethopteris virginiana leaves room for doubt. Since Fontaine and White's specimens are no longer available (W.H. Gillespie, pers. comm.), these doubts cannot be dispelled by direct observation. Indeed, it is unclear whether the specimens figured on Fontaine \& White's (1880) pl. XXXII really belong to the same species as those illustrated on their pl. XXXIII, figs 1-4a. There is at least one specimen on their pl. XXXII, fig. 2 which almost certainly represents a pecopterid fern, and it may well be that the entire plate (pl. XXXII, figs 1-5) belongs with the pecopterids. Fontaine and White's nervation diagram (pl. XXXII, fig. 1a) suggests that Polymorphopteris subelegans (Potonié, 1893) Wagner, 1959 may be involved. The Alethopteris virginiana, as meant in Fontaine \& White's description (1880: 88-89), seems to be represented by the specimens figured on their pl. XXXIII, figs 1-4a. One of the present writers (RHW) has had the privilege of being allowed to collect from the shale parting (Cassville Shale) in the Waynesburg Coal, under guidance from W.H. Gillespie. The remains recovered from this locality (Fontaine \& White's type locality) include an Alethopteris which is the same as Alethopteris leonensis. If these remains are identified with Alethopteris virginiana pars, this may be regarded as the senior synonym, although a neotype will have to be designated; a doubtful procedure if Fontaine \& White's concept of their species is to be identified correctly.
The three species, Alethopteris ambigua, Alethopteris lesquereuxii, and Alethopteris leonensis (= Alethopteris virginiana pro parte?) are certainly comparable, though probably not identical. It is noted that Zodrow \& Cleal (1998) overlooked the illustration of specimens attributed to Alethopteris ambigua var. gibsonii Lesquereux, 1879 from Point Aconi Seam in the Sydney Basin by Wagner (1968: 39-40, pl. 4, figs 15-18a). These specimens were described separately from undoubted Alethopteris ambigua. They are quite comparable to the specimens illustrated from the Hub and Upper Bonar seams by Zodrow \& Cleal (1998). Perhaps, the Point Aconi specimens which were attributed to Alethopteris ambigua var. gibsonii by Wagner (1968), should be referred to Alethopteris lesquereuxii. Indeed, their slightly higher vein density would point in this direction. Earlier illustrations under the name of Alethopteris friedelii by Bell (1938), also from the Sydney Coalfield, are shown at natural size and cannot be judged very well from the illustration. These were included in the synonymy of Alethopteris ambigua by Wagner (1968), but this may have to be revised, at least in part. (Attention is drawn to the strong similarity between Bell's pl. LXII, fig. 2 and Zodrow \& Cleal's pl. 8, fig. 1.)

Zodrow \& Cleal (1998: pl. 2, figs 1-3) figured side by side a pinna of Alethopteris lonchitica (lectotype from Saarland) and a much enlarged specimen showing several fragmentary pinnules from the Sydney Basin in Nova Scotia which shows a vein density that is at least three times higher. (Note the very different enlargements for these two specimens.) The pinnules from the Sydney Basin seem to belong to the Alethopteris lonchitifolia-missouriensiswestphalensis complex (as Zodrow and Cleal do, in fact, assume), and cannot be attributed to Alethopteris lonchitica.

Since Alethopteris lonchitica is here regarded as identical with Alethopteris friedelii (= Alethopteris ambigua sensu Wagner, 1968, non Zodrow \& Cleal, 1998), the specimens attributed to Schlotheim's species in the literature are practically all to be reassigned. No attempt was made by Zodrow \& Cleal (1998) to revise the vast amount of literature in which Alethopteris lonchitica has been named. This is understandable because they described material from the upper Westphalian (of the Sydney Basin in Nova Scotia), whereas most of the records of Alethopteris lonchitica (auctorum) correspond to upper Namurian/lower Westphalian specimens. In the Maritime Provinces of Canada this involves material from the Cumberland Basin (near the Bay of Fundy in Nova Scotia) and coeval strata in the area of Saint John (New Brunswick). Specimens from these areas were recorded as Alethopteris lonchitica by Stopes (1914) and Bell (1944, 1966). Both authors admitted Alethopteris discrepans as a synonym. This is a species described from the "Fern Ledges" at Saint John. (N.B. Dawson's type specimens are very fragmentary.)

Attention is drawn to Alethopteris lancifolia, a species with relatively large pinnules showing a dense nervation 


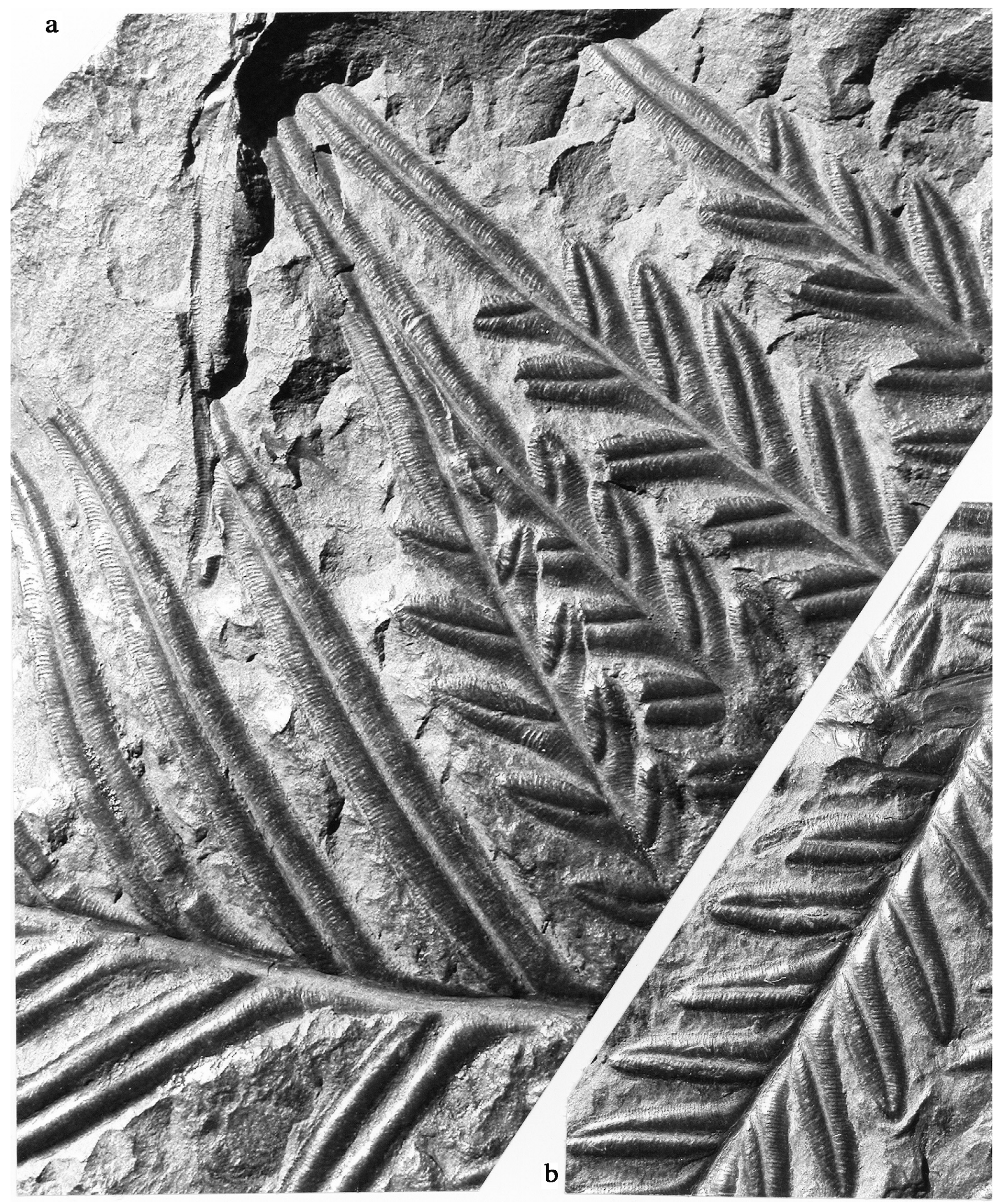

Figure 3. a, Enlargement (x 3) of part of the smaller penultimate pinna fragment of holotype (Fig. 2) showing the transition between last order pinna with elongate terminals and almost parallel-sided elongate pinnules in near-terminal position. b, A single pinna of holotype with average size lateral pinnules (x 3). N.B. Note compression borders. Repository: Palaeontology Unit, British Geological Survey, Keyworth. 
similar to that of Alethopteris lonchitica sensu Zeiller (see Buisine, 1961). This species, originally described from lower Westphalian strata in South Limburg in the Netherlands (Wagner, 1961), has most recently been recorded from the "Fern Ledges", New Brunswick (Wagner, 2005). It is unclear whether or not this species should be included in the range of variation of Alethopteris urophyl$l a$. The present writers provisionally exclude this species on the basis of its relatively broader pinnules of generally larger size.

Another species which has been recorded in the literature with specimens comparable to Alethopteris lonchitica (auctorum) is Alethopteris decurrens. It appears that the range of variation admitted for Alethopteris decurrens in the literature may be excessive and that material of Alethopteris lonchitica (auctorum) has been included. These more doubtful records will be analysed in the present paper.

It may be that Alethopteris heterophylla (Lindley \& Hutton, 1832) Göppert, 1836, from Felling Colliery in Northumberland, England, is the same as Alethopteris urophylla and Alethopteris lonchitica (auctorum). This assumption is reflected in Kidston (1886: 133) and in the Catalogue of the Hutton Collection published by Howse in 1888, where Pecopteris heterophylla appears as a synonym of Alethopteris lonchitica. However, Crookall (1955: 26) regarded Lindley \& Hutton's species as a synonym of Alethopteris decurrens, a similar species to Alethopteris urophylla, with generally more slender pinnules showing a wider, less regular venation. This may be the correct identification, although some doubt remains. It is noted that another one of the synonyms quoted by Howse is Alethopteris urophylla. Lindley and Hutton's description of Alethopteris heterophylla predates Brongniart's Alethopteris urophylla, and if these two species should prove to be identical, it is Lindley and Hutton's epithet which may be the correct one for the mainly lower Westphalian species recorded generally as Alethopteris lonchitica (auctorum). Unfortunately, the type of Pecopteris heterophylla is reputedly lost (McLean - fide C.J. Cleal, pers. comm. 17-10-2006), and this leaves Alethopteris urophylla as the more reasonable choice for typifying the morphospecies recorded in the literature as Alethopteris lonchitica (auctorum). Lindley and Hutton's illustrations are diagrammatic drawings which leave ample room for speculation. It is also noted that the slender pinnules depicted suggest Alethopteris decurrens rather than Alethopteris urophylla.

\section{COMMENTS ON THE IDENTITY OF Alethopteris lonchitica SCHLOTHEIM EX STERNBERG}

Although the type specimen is in the Museum für Naturkunde, Berlin, Schlotheim's species was validated by
Sternberg (1825). It thus merited inclusion in the Catalogue published by Kvaček \& Straková (1997), who noted that the drawing produced by Schlotheim (1804) contained elements from two different sides of the same specimen, an ironstone nodule ("Sphaerosiderit" - fide M. Barthel, pers. comm. 20-06-2008). Altogether, fragments of 8 different pinnae of the last order were illustrated, among which Kvaček and Straková selected as the lectotype a fragment on the right hand side of the illustration (which occurs as a single fragment on one face of the rock specimen). This is the specimen illustrated photographically by Zodrow \& Cleal (1998) on their pl. 2, figs 1-2. It represents a pinna fragment which apparently terminates in a large apical pinnule. This is how it was depicted by Schlotheim (1804: Taf. XI, fig. 22). However, the photograph is less clear in this respect, and a re-examination of the specimen by $\mathrm{M}$. Barthel, at the present writers' request, has shown that organic conection cannot be proved. The near-terminal part of the pinna is damaged, and it is noted that the apparent apical pinnule closely resembles a standard lateral pinnule. A fortuitous superposition belongs to the possibilities. Its size and shape does not fit for any of the upper Westphalian species of Alethopteris from Saarland. Unfortunately, the lectotype does not lend itself to preparation (M. Barthel, pers. comm.).

Zodrow \& Cleal (1998) illustrated photographically the various pinna fragments of Schlotheim's specimen from Saarland, but they did not provide a description. Instead, specimens from the Sydney Coalfield in Nova Scotia were described on the assumption that these would coincide with Schlotheim's species. This seems questionable. Their pl. 2, fig. 3 (same specimen as in Bell, 1938: pl. LXI, fig. 5 - as Alethopteris lonchitica) shows several pinnule fragments with a higher vein density than the lectotype. Although quite fragmentary, it seems likely that this specimen belongs to the Alethopteris lonchitifolia-missouriensis-westphalensis complex. Zodrow \& Cleal (1998: 72) indeed assumed that Alethopteris lonchitifolia would be the same as Alethopteris lonchitica, thus accepting without question the opinion expressed by Thomas \& Cleal (1993a: 115). They did not consider any other alternative, such as Alethopteris friedelii (= Alethopteris pseudoaquilina Potonié, 1893) which they regarded as a probable synonym of Alethopteris grandinii (Brongniart, 1828) Göppert, 1836 (see Zodrow \& Cleal, 1998: 96).

The assumption that Alethopteris lonchitica would be identifiable with Alethopteris lonchitifolia has led to a description by Zodrow and Cleal which is only applicable to the Alethopteris lonchitifolia-missouriensis-westphalensis complex. This is unfortunate, because it seems likely that the type Alethopteris lonchitica belongs to the same species as Alethopteris ambigua (= Alethopteris pseudoaquilina, Alethopteris friedelii). The problem is compounded by an emended diagnosis for Alethopteris ambigua as proposed by Zodrow \& Cleal (1998: 97) who incorporated, 
albeit tentatively, Alethopteris lesquereuxii, a similar but not identical species. Indeed, Zodrow \& Cleal (1998) illustrated specimens of Alethopteris lesquereuxii from the Sydney Basin in Nova Scotia as Alethopteris ambigua. This is similar material to that figured by Wagner (1968) as Alethopteris ambigua var. gibsonii, also from the Sydney Basin.

It is here concluded that Alethopteris lonchitica, as typified by Schlotheim's type specimen from Saarland, is the same as Alethopteris ambigua (synonyms: Alethopteris pseudoaquilina, Alethopteris friedelii), a widespread element of upper Westphalian floras. The similar species, Alethopteris lesquereuxii, is characterised by generally longer pinnules with a more regular venation. It is agreed with Zodrow \& Cleal, that the difference is slight (as is apparent by the inclusion of Alethopteris ambigua var. gibsonii as figured by Wagner, 1968, in Alethopteris lesquereuxii). The proper identity of Alethopteris gibsonii, as fig- ured and described by Lesquereux (1879-80), can only be established by a re-examination of Lesquereux's material. His diagrammatic drawings do not provide an adequate basis for discrimination.

\section{SYSTEMATIC DESCRIPTION}

The suprageneric taxonomy used in the present paper follows that of Cleal (1993) in "The Fossil Record 2". In the list of synonymy the following system of annotations is used (shortened/simplified from Cleal \& Shute, 1995): * The protologue; $\S$ First publication of currently accepted combination; ? References to doubtful specimens due to poor illustration or preservation; $p$ pars: only part of the specimens published belong to the species; $\mathrm{v}$ vide: the authors have seen the specimen(s).

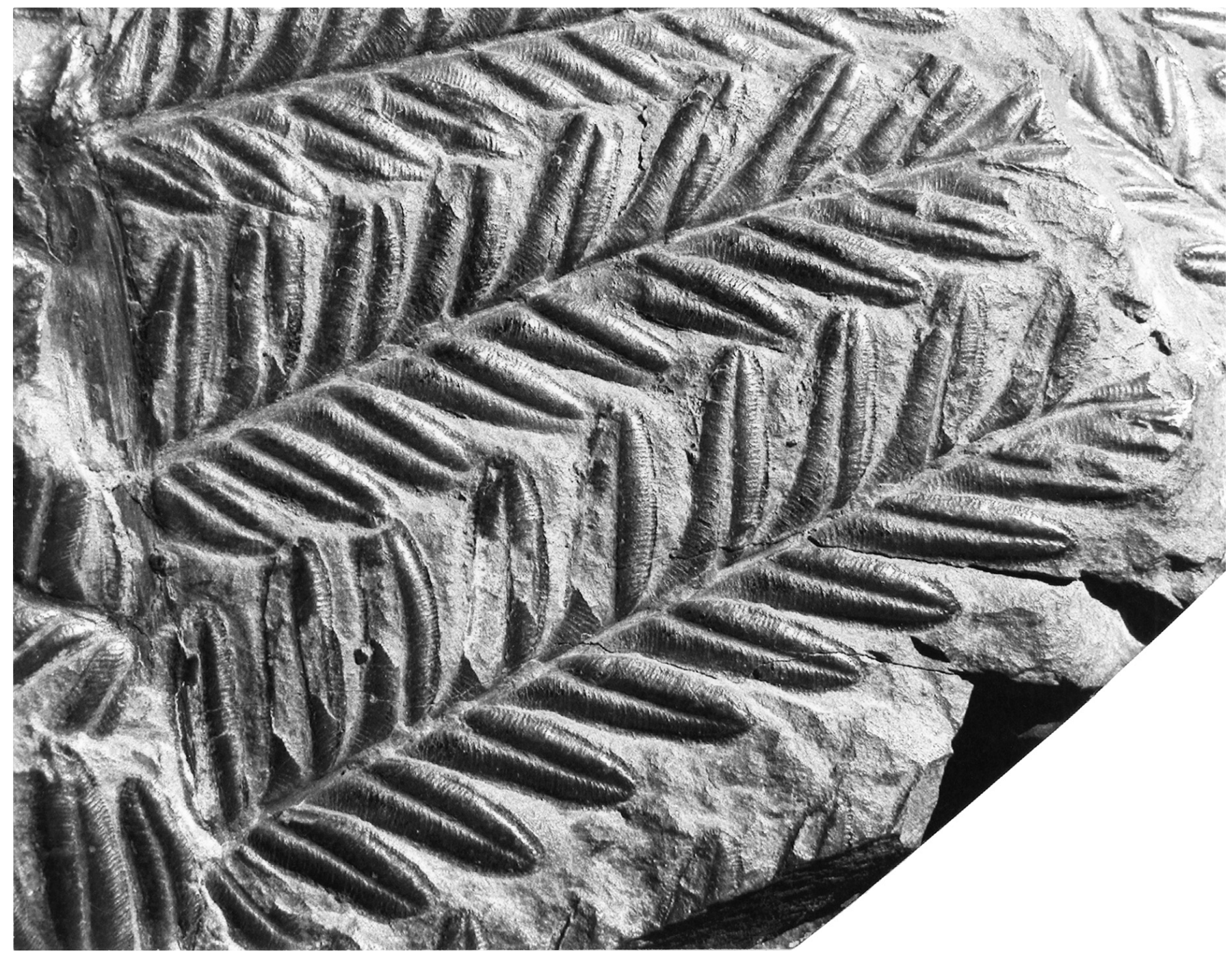

Figure 4. Enlargement (x 3) of part of the larger penultimate pinna of holotype, showing average size lateral pinnules. These are narrowly confluent, asymmetrical due to acroscopic constriction, and nearly biconvex, with compression borders. Repository: Palaeontology Unit, British Geological Survey, Keyworth. 
Class CYCADOPSIDA Barnard \& Long, 1975 Order TRIGONOCARPALES Seward, 1917 emend. Meyen, 1984

Family Trigonocarpaceae Seward, 1917 emend. Meyen, 1984

Genus Alethopteris Sternberg, 1825 emend. Zodrow \& Cleal, 1998

Type species: Alethopteris lonchitica Schlotheim, 1820 ex Sternberg, 1825

This genus of pteridosperm fronds has been discussed recently by Zodrow \& Cleal (1998), who gave the fol- lowing emended diagnosis: "Bipartite fronds, which may attain large dimensions, up to at least 7 metres long. Primary pinna branches usually tripinnate, with no intercalated pinnules or pinnae on the primary rachis branches or secondary rachises. Rachises usually striate. Pinnules strongly asymmetric, fused at the base, decurrent at the basiscopic side, straight or slightly constricted at the acroscopic side. Pinnule lamina generally rather thick, giving a vaulted aspect to the pinnules. Lobing may be rather abrupt or more gradual, but lobing parts of the frond never seem to predominate. Venation characterized by a well-marked and strongly decurrent midvein and numerous, non-anastomosed laterals that meet the pinnule margin at about right-angles or somewhat obliquely. The

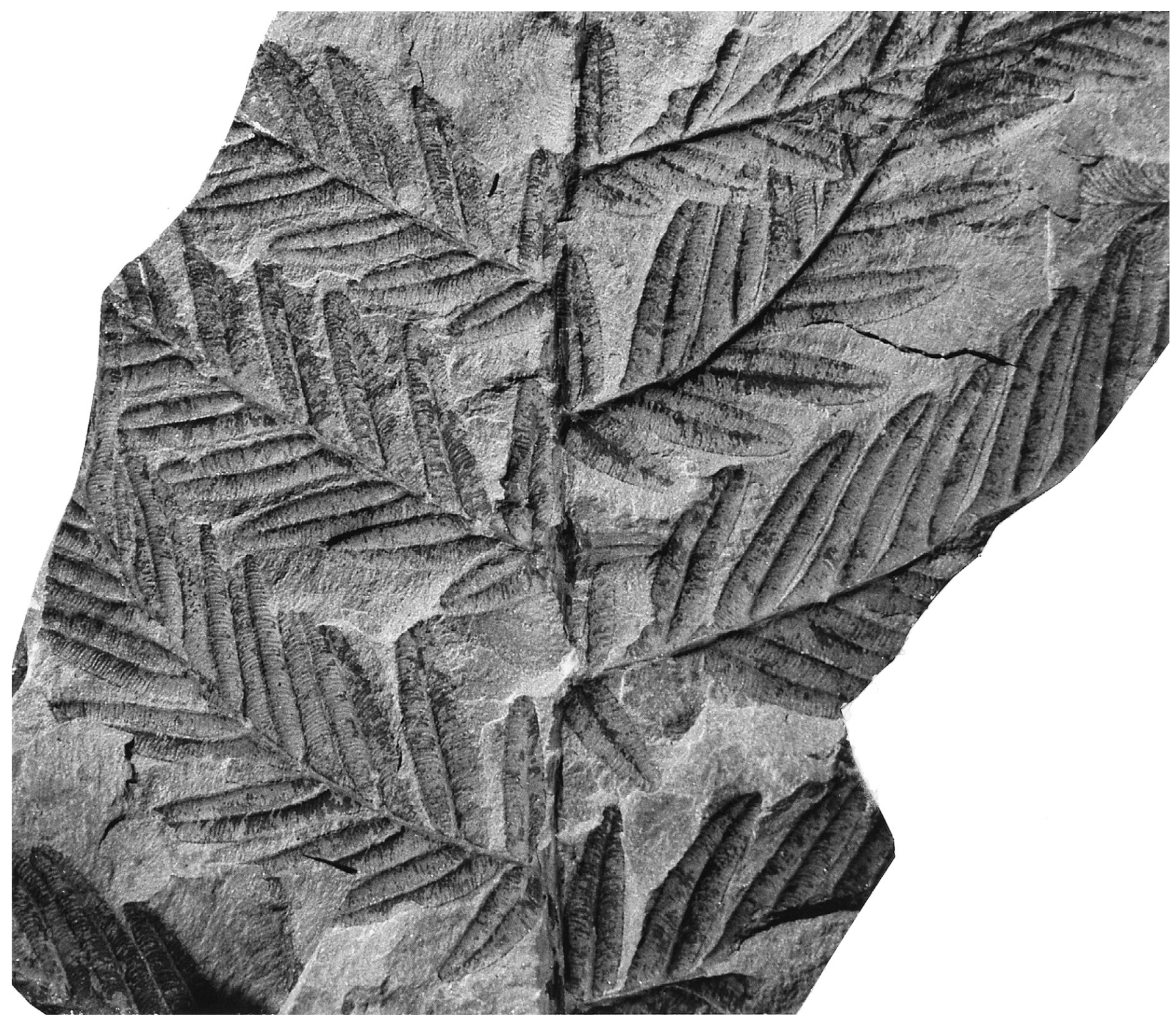

Figure 5. Middle part of a pinna of the penultimate order (x 3), showing relatively large lateral pinnules, which are very narrowly confluent. Origin: Barnsley Seam, Yorkshire, England, lower Duckmantian (loc. 2872). Collected by C.J. Cleal, 1974, from opencast site south of Barnsley. Repository: Centro Paleobotánico, Córdoba. 
lateral veins fork at irregular intervals, mostly one time, sometimes by a tripartite division, and occasionally each fork divides again (forking twice). Pinnules hypostomatic. Adaxial epidermis shows little marked differentiation of the cells, except over the midvein. Abaxial epidermis has clearly differentiated cells between the costal and intercostal fields. Stomata restricted to the intercostal fields of the abaxial cuticle are anomocytic, or have only a weakly developed, single ring of subsidiary cells. Trichomes are restricted to the abaxial surface and the midveins."

The size of Alethopteris fronds seems to have been very substantial, as follows from the fragment (less than half the frond) of $1.20 \mathrm{~m}$ length recorded by Laveine (1986), who calculated a total frond size of c. $7 \mathrm{~m}$.

The genus Alethopteris, as defined above in its restricted sense, does not include Macralethopteris Jongmans \& Gothan, 1935, a genus which is virtually restricted to its type species, Macralethopteris hallei Jongmans \& Gothan, 1935 (see comments in Wagner, 1968: 21-22). This is an East Asian (Cathaysian) species of Permian age. It is noted that several species which have been attributed to Alethopteris in the Permian floras of East Asia, China in particular, are subject to reservation. In some cases the genus Callipteridium Weiss, 1870, is involved; in other cases the affinities are unclear. Alethopteris seems to be essentially Pennsylvanian in age, although recorded also in upper Mississippian strata and extending into lowermost Permian. It is fairly closely circumscribed morphologically. It forms a natural unit with Lonchopteridium (Gothan, 1909 - as a subgroup of Lonchopteris) Bode, 1941 (as a separate genus), and Lonchopteris Brongniart, 1828a. The little evidence that is available on reproductive organs, suggests that Neuralethopteris Cremer, 1893 also belongs to the same natural unit (compare Buisine, 1961; Goubet et al., 2000).

The type species, Alethopteris lonchitica, has given rise to taxonomic problems as detailed in the present paper.

\section{Alethopteris urophylla (Brongniart, 1834) Göppert, 1836 \\ Figs 1-12}

?* 1832 Pecopteris heterophylla Lindley \& Hutton, 113-114, pl. 38, figs 1-2 (type specimen unavailable and presumably lost).

p 1833 Pecopteris lonchitica; Brongniart, pl. 84, figs 1, 1A (copied by Hofmann \& Ryba, 1899, Taf. VIII, figs 1, 1a); non pl. 84, figs 2-7 (= Neuralethopteris neuropteroides (Šusta, 1927) Josten, 1983); non pl. 128, both figures (which may be Alethopteris friedelii = Alethopteris lonchitica - also according to Buisine, 1961: 109).

* v 1834 Pecopteris urophylla Brongniart, 290-291, pl. 86 (refigured in the present paper as Figs 1-4).

1835 Pecopteris lonchitica; Lindley \& Hutton, 197-199, pl. 153, figs 1-2.
? 1836 Alethopteris heterophylla (Lindley \& Hutton) Göppert, 297.

$\S 1836$ Alethopteris urophylla (Brongniart) Göppert, 300.

p 1848 Pecopteris lonchitica; Sauveur, pl. XL, fig. 3; pl. XLII, fig. 4 (?) (may be either Alethopteris urophylla or Alethopteris decurrens); non pl. XLI, figs 1, 2 (= Neuralethopteris schlehanii (Stur, 1877) Cremer, 1893); non pl. XLII, fig 5 (= Neuralethopteris sp. indet.).

* 1848 Pecopteris multiformis Sauveur, Pl. XXXVI, fig. 1 (placed in synonymy with Alethopteris lonchitica by Kidston, 1886: 134).

* 1862 Pecopteris (Alethopteris) decurrens sp. nov. (non Artis); Dawson, 322, pl. XV, figs 40a-c [diagrammatic drawings of very fragmentary specimens; the homonym Pecopteris (Alethopteris) decurrens was changed to Pecopteris discrepans by Dawson in 1863; Stopes, 1914, referred Pecopteris (Alethopteris) discrepans to Alethopteris lonchitica, meaning Alethopteris lonchitica auctorum].

* 1862 Pecopteris (Alethopteris) ingens sp. nov.; Dawson, 322 , pl. XV, figs 41a, b [drawings of fragmentary pinnules which probably belong to Alethopteris lonchitica (auctorum) - see the exhaustive comments by Stopes, 1914: 95-96].

* 1863 Pecopteris discrepans Dawson, 468 (name change on the basis of homonymy with Pecopteris decurrens Lesquereux - see Lesquereux in Rogers, 1858; however, the real homonym is Filicites decurrens = Alethopteris decurrens).

1865 Alethopteris discrepans Dawson; Dawson in Hartt in Bailey's Report, 136-137.

1868 Alethopteris discrepans Dawson; Dawson, 552-553, fig. 192 I (copy of the drawing in Dawson, 1862: pl. XV, fig. 40a).

p 1868 Alethopteris lonchitidis Sternberg (as Alethopteris lonchitides in plate explanations); Roehl, Taf. XIV, figs 2, 4, figs 1, 3, 3a (?); non Taf. XXXI, figs 4, 4a (= Neuralethopteris sp. indet.).

1868 Alethopteris urophylla Goeppert (sic); Roehl, 75, Taf. XXII, fig. 7.

p 1868 Alethopteris heterophylla Goeppert (sic); Roehl, Taf. XXXII, figs 2, 9; non Taf. XXXII, figs 5A,B, 5Ba (could be Neuralethopteris schlehanii) (Kidston, 1886: 133, placed all these figures in Alethopteris lonchitica). Alethopteris discrepans Dawson; Dawson, 54, pl. XVIII, fig. 204 (specimen refigured photographically by Stopes, 1914, pl. XIII, fig. 3); non fig. 203 ? (possibly Alethopteris lonchitifolia?); non fig. 205? [cannot be judged properly; nervation as drawn by Dawson resembles that of Neuropteris obliqua (Brongniart, 1834) Göppert, 1848].

1886-88 Alethopteris lonchitica Schlotheim; Zeiller, 225228, pl. XXXI, figs 1, 1A (reproduced in Gothan \& Franke, 1929, Taf. 12; Zeiller's figures are partially reproduced in Ralli, 1933: pl. XVI, figs 3, 3a).

1899 Alethopteris lonchitica Brongniart; Hoffman \& Ryba, Taf. VIII, figs 1, 1a (same as Brongniart, 1833: pl. 84, figs 1, 1A).

1901 Alethopteris lonchitica Schlotheim; Kidston, 195, pl. XXXII, figs 2, 3 . 
1907a Alethopteris lonchitica Schlotheim; Zalessky, 397398, Tab. XVI, fig. 6 [Zalessky \& Tchirkova, 1938 , included this specimen in their synonymy of Alethopteris davreuxii (Brongniart, 1828) Göppert, 1836].

1907a Alethopteris decurrens Artis; Zalessky, Tab. XVI, fig. 5 (only cited in the plate explanation).

1907b Alethopteris decurrens Artis; Zalessky, 467-468, Tab. XVIII, figs 1, 4.

1907b Alethopteris Serli Brongniart; Zalessky, 469-470, Tab. XXI, fig. 2 (included by Zalessky \& Tchirkova, 1938, in Alethopteris lonchitica - erroneously cited as fig. 1).

p 1910 Johannophyton discrepans n. gen. Dawson sp.; Matthew, 83, pl. III, figs 1, 2, 4 (copies of Dawson, 1871: pl. XVIII, figs 203-205), figs 3, 5 (diagrammatic drawings), fig. 6 (diagrammatic drawing), fig. 9; non figs 8, 10 (sporangia); non pl. II, fig. 7 (= Alethopteris lancifolia acc. to Wagner, 2005: 16); non figs 8-9 (sporangia)

1910 Alethopteris lonchitica Schlotheim; Seward, 553, 574, fig. 290A (after Zeiller, 1886: pl. XXXI, fig. 1A), fig. 364A.

1913a Alethopteris lonchitica; Franke in Potonié, 161, Fig. 1, Fig. 2 (after Zeiller, 1886: pl. XXXI, fig. 1), Fig. 3 (drawing).

1913 Alethopteris lonchitica Schlotheim; Gothan, 175177, Taf. 39, fig. 1 (after Franke in Potonié, 1913a: Fig. 1); Taf. 40, fig. 3 (diagrammatic drawing of a pinnule from the specimen figured on Taf. 39, fig. 1).

1913-14 Alethopteris Serlii Brongniart; Bureau, 322-323 (excluding synonymy), pl. LXXIX, fig. 6.

p 1914 Alethopteris lonchitica Schlotheim (= Alethopteris discrepans Dawson); Stopes, 47-53, pl. XII, fig. 30, pl. XIII, fig. 31 (photographic illustration of the specimen figured by Dawson, 1871: pl. XVIII, fig. 240), figs 32, 33 (rather poorly preserved fragments which cannot be judged adequately from the illustration); non pl. XXII, fig. 57A (pinna fragment with widely confluent pinnules of Alethopteris sp. indet.); textfigs 8A-C (rather diagrammatic drawings); non pl. XVIII, fig. 46 (sporangia, and pinnule fragments which may belong to either Alethopteris sp. or Neuralethopteris sp.).

1915 Alethopteris Serli Brongniart; Gothan \& Jongmans in Jongmans, 173, Taf. V, fig. 1.

1916 Alethopteris lonchitica Schlotheim; Kidston, pl. XIII, fig. 6.

1923 Alethopteris lonchitica; Scott, 172, fig. 69 (although reduced in size and poorly figured, it seems referable to Alethopteris urophylla).

1928 Alethopteris lonchitica; Jongmans, 15, Plaat 7, fig. 1. 1928 Alethopteris lonchitica Schlotheim; Susta, 439, Taf. XXXIV, Abb. 3, Taf. XXXV, Abb. 3 [as Alethopteris lonchitica (valida?) in the plate explanation].
1996 acc. to Šimůnek, 1996); non Taf. XXXV, Abb. 5 (may also be referred to Alethopteris havlenae). Alethopteris species; de Voogd, 23, Taf. III, fig. 19.

Alethopteris lonchitica (Schlotheim); Crookall, p. 58, pl. XXX, fig. e.

Alethopteris lonchitica; Jongmans, 9, 13, Fig. 32.

Alethopteris lonchitica Zeiller; Corsin, 18, pl. VIII, figs 1, 1a; texte-fig. 7 .

Alethopteris lonchitica (Schlotheim); Renier \& Stockmans in Renier et al., 85, pl. 75.

Alethopteris lonchitica (Schlotheim); Zalessky \& Tchirkova, 46-47, 157, fig. 54.

Alethopteris lonchitica Schlotheim; Jongmans, Taf. XXIII, figs 57-59; Taf. XXIV, fig. 64; Taf. XXV, fig. 69.

Alethopteris Helenae Lesquereux; Arnold, p. 188189, pl. XIX, figs 5, 6.

Alethopteris decurrens; Arnold, pl. XIX, fig. 7; non pl. XIX, fig. 4 (pinna terminal).

Alethopteris lonchitica (Schlotheim); Stockmans \& Willière, pl. C, figs 6, 6a.

Alethopteris lonchitica (Schlotheim) Göppert; Novik, Tab. LVII, figs 7, 8 (poorly figured and difficult to judge).

p 1952-53 Alethopteris lonchitica (Schlotheim); Stockmans \& Willière, 239-240, pl. XXVIII, figs 14, 14a (after Stockmans \& Willière, 1951: pl. C, figs 6, 6a), pl. L, fig. 7; non pl. L, figs 8-10 (= Alethopteris $\mathrm{cf}$. decurrens); non pl. LII, figs 6-9 (too fragmentary to be judged properly).

1952-53 cf. Aulacotheca Idelbergeri Halle; Stockmans \& Willière, pl. L, fig. 6 (pinna fragment figured alongside Aulacotheca synangium).

p 1953 Alethopteris lonchitica (Schlotheim) Unger incl. f. Serli (Brongniart); Gothan, 16-18, Taf. 4, fig. 2, fig. 5 (previously published as a drawing by Franke in Potonié, 1913a: Fig. 3, and Gothan \& Franke, 1929: Abb. 9); Taf. 5, figs 1, 4, 5; Taf. 6, figs 2-4; non Taf. 4, fig. 1 (= Alethopteris lancifolia acc. to Wagner, 1961, 1968, and Wagner, 2005), fig. 3 (= Alethopteris lancifolia?), fig. 4 (as f. Serli; = Alethopteris westphalensis acc. to Wagner, 1968: 154); non Taf. 5, figs 2, 2a (to be compared with Alethopteris westphalensis), fig. 3 (= Alethopteris cf. grandinioides Kessler, 1916 = Alethopteris pseudograndinioides Zodrow \& Cleal, 1998); non Taf. 6, fig. 1 (as f. Serli; possibly Alethopteris westphalensis acc. to Wagner, 1968: 154 - previously figured as a drawing by Franke, 1912 and Franke in Potonié, 1913b as Alethopteris serli forma platyrachis n.f.).

1953a Alethopteris lonchitica Schlotheim; Jongmans, 24, pl. 8, figs 47-49a.

? 1954 Alethopteris lonchitica (Schlotheim) Göppert; Novik, Tab. XIX, fig. 5 (difficult to judge from the figuration).

p 1955 Alethopteris lonchitica Schlotheim; Crookall, 22-26 (excluding synonymy), pl. V, fig. 2; pl. X, fig. 1 (the complete specimen, partially figured at $\mathrm{x} 1 / 2$ size in Seward's text-book, 1910: fig. 364A), fig. 3; textAbb. 1, 2; non Taf. XXXIV, Abb. 1 (= Neuralethopteris?), Abb. 5 (to Alethopteris havlenae Šimůnek, 
fig. 14H; non pl. V, fig. 1 (= Alethopteris lancifolia acc. to Wagner, 1961, 1968, and Wagner, 2005); non text-fig. 7 (copy of original figure of Filicites lonchiticus).

1957 Alethopteris lonchitica Schlotheim; Gothan \& Remy, 118-119, Abb. 110 (after Franke in Potonié, 1913a, and Gothan, 1953: Taf. 4, fig. 5), Abb. 111.

1957 Alethopteris lonchitica Schlotheim; Purkyňová, Tab. III, figs 3, 6 .

? 1957 Alethopteris lonchitica (Schlotheim) Zeiller; Stopa, 83-84, 191, pl. XXXI, fig. 7 (difficult to judge from the photograph).

1958 Alethopteris lonchitica (Schlotheim); Stockmans \& Willière, pl. IV, figs 2-3.

p $\quad 1961$

Alethopteris lonchitica (Schlotheim) Zeiller; Buisine, 99-115, pl. XIII, fig. 1 (refigured in Figs 8-10 of the present paper); pl. XIII, figs 2-2b; pls XIVXVI; pl. XVII, fig. 2, 4; pl. XVIII, figs 1-1b; pls XIX, XX; text-figs 9a-c; non pl. XVII, figs 1, 3 (= Alethopteris densinervosa); non pl. XVIII, fig. 2 (= Alethopteris densinervosa).

p 1961 Alethopteris serli Brongniart; Buisine, pl. VIII, figs 2, 2a (= Alethopteris urophylla acc. to Wagner 1968: 59, 139); non pls I-VII, pl. VIII, figs 1, 1a (= Alethopteris densinervosa acc. to Wagner, 1968: 59); non pl. IX, figs 1, 1a (= Alethopteris westphalensis acc. to Wagner, 1968); non pl. X, figs 1, 1a, 3-4 (= Alethopteris densinervosa acc. to Wagner, 1968); non pl. IX, figs 1, 1a, pl. X, figs 2, 2a, pl. XI, figs 1-2, pl. XII, figs 1a-1c (= Alethopteris westphalensis acc. to Wagner, 1968).

1961 Alethopteris lonchitica (Schlotheim) Unger (sic); Josten, Taf. 10, Fig. 4.

p 1961 Aulacotheca hallei Hemingway; Stockmans \& Willière, pl. IX, fig. 12 (pinna fragment illustrated alongside Aulacotheca synangium).

1962 Alethopteris lonchitica Schlotheim; Purkyňová, 7576, 113, fig. 41b, Tab. XX, fig. 5 .

1964 Alethopteris lonchitica (Schlotheim) Unger (sic); Drägert, 40, Taf. 2, fig. 3.

p 1965 Alethopteris lonchitica (Schlotheim); Stockmans \& Willière, Pl. V, figs 3-3a (fragmentary); non figs 4, 4a (= Alethopteris havlenae).

1966 Alethopteris decurrens Artis; Migier, 85, 89, Tab. VIII, figs 3-4.

v 1966 Alethopteris lonchitica (Schlotheim) Göppert; Bell, pl. VII, fig. 4.

p 1969 Alethopteris sp.; Daber, 260, Taf. IX, Bild 7; non Taf. I, Bild 1 (cannot be judged from the photograph).

p 1970 Alethopteris lonchitica (Schlotheim) f. typica Gothan; Havlena, pl. I, figs 10, 11; non pl. I, fig. 12 (too fragmentary to be judged properly).

1971 Alethopteris lonchitica Schlotheim; Purkyňová, 141, Tab. VII, figs 1-2.

p 1972 Alethopteris lonchitica Schlotheim; Migier, 145, 157, Tab. XIV, fig. 4; non Tab. XIII, fig. 5 (? - cannot be judged properly from the illustration).

1973 Alethopteris serli Brongniart; Biţoianu, pl. II, fig. 13.

1974 Neuralethopteris jongmansi Laveine; Fiebig \& Leggewie, Taf. 4, fig. 7.
1975 Alethopteris decurrens Artis; van Amerom \& Lambrecht, p. 153, 156, Taf. VI, figs 5a, b.

1975 Alethopteris lonchitica (Schlotheim) Sternberg; van Amerom, pl. 1, figs 4, 5; pl. 1, figs c, d (diagrammatic drawings)

1977 Alethopteris lonchitica (Schlotheim) Zeiller; Purkyňová, 291, 295, pl. IV, fig. 2 (after Purkyňová, 1971: Tab. VII, figs 1, 1a).

1977 Alethopteris lonchitica (Schlotheim) Sternberg; Remy \& Remy, 272-273, Bild 149a (after Gothan \& Remy, 1957: Abb. 111), Bild 149b (after Gothan \& Remy, 1957: Abb. 110), Bild 149c (nervation diagram).

1977 Alethopteris lonchitica auct. (non Schlotheim?); Scott, 461, pl. 51, fig. 1 (refigured as Alethopteris sp. in Scott \& Smith, 1977, pl. 2, fig. 3, figs 2, 3, $11,12)$.

? 1977 Alethopteris lonchitica (Schlotheim); Tenčov, 5556, Taf. XVIII, Bild 5.

v 1981 Alethopteris urophylla (Brongniart); Wagner in Mamet \& Martínez, 110.

v 1983 Alethopteris lonchitica (Von Schlotheim) Zeiller; Wagner, 157.

v 1983 Alethopteris urophylla (Brongniart) Von Roehl (sic); Wagner, 157.

v 1983 Alethopteris cf. michauxi Buisine; Wagner in Wagner \& Bowman, 154.

1983 Alethopteris lonchitica (Schlotheim) Sternberg; Josten, 128-129, Taf. 47, figs 1, 1a; Abb. 91 (nervation diagram).

p 1983 Alethopteris valida Boulay; Josten, 132, Abb. 95; Taf. 48, figs 2, 2a; non Taf. 48, figs 1, 1a (=Alethopteris valida Boulay, 1876).

p 1984 Alethopteris lonchitica (Schlotheim) Zeiller; Havlena, 371-372, pl. I, fig. 1 (same as in Havlena, 1970: pl. I, figs 10, 11); pl. II, fig. 1, figs 4, 5 (? - also to be compared with Alethopteris davreuxii); pl. III, figs 1-3, 7-9; pl. IV, figs 1, 4, 5; non pl. III, fig. 6 (? - fragmentary; same as in Havlena, 1970: pl. I, fig. 12).

v 1984 Alethopteris cf. michauxi Buisine; Wagner in Wagner et al., 35 (same as in Wagner \& Bowman, 1983).

1985 Alethopteris lonchitica (Schlotheim); Delvolvé \& Laveine, 293-294, pl. A, fig. 2, pl. 6, fig. 4.

p 1985 Alethopteris lonchitica (Schlotheim) Sternberg; Gillespie \& Rheams, 194, 195, pl. II, fig. 2; non pl. I, fig. 3 (to be compared with Alethopteris valida).

1989 Alethopteris lonchitica (Schlotheim) Sternberg; Gillespie et al., 5, pl. 2, fig. 7.

1990 Alethopteris lonchitica Schlotheim; Purkyňová, 219220, Tab. II, fig. 4, Tab. II, fig. 5 (after Purkyňová, 1962: Tab. XX, fig. 5).

1991 Alethopteris lonchitica (Schlotheim) Sternberg; Josten, 291-293, Taf. 158, figs 1, 1a, Taf. 159.

1993a Alethopteris urophylla (Brongniart) Presl in Sternberg; Thomas \& Cleal, 115, Fig. 4A (same as in Thomas \& Cleal, 1993b: 21, left figure); Figs 4B, 5A.

1994 Alethopteris urophylla; Cleal \& Thomas, 125, pl. 20 (the same specimen as figured in Thomas \& Cleal, 1993a and 1993b), text-fig. 61A (after Crookall, 1955). 
1995 Alethopteris lonchitica Brongniart non von Schlotheim; Álvarez-Vázquez, 57-59, lám. 14, figs 1-3; lám. 15, figs $1 \mathrm{a}, 1 \mathrm{~b}$.

1995 Alethopteris lonchitica Schlotheim; BrousmicheDelcambre, Mercier \& Coquel, 336, 338, 339, photo 5,6 .

1995 Alethopteris lonchitica (Schlotheim) Zeiller; Kotasowa in Dybová-Jachowicz et al., pl. IV, fig. 2.

1995 Alethopteris lonchitica (Schlotheim) Sternberg (with cf. in the text); Schultka, 24, Taf. 27, figs 1-3.

1995 Alethopteris tectensis Stockmans \& Willière; Schultka, 24, Taf. 27, figs 4, 5.

1995 Alethopteris cf. lancifolia Wagner; Schultka, 24, Taf. 27, fig. 6.

? 1995 Alethopteris cf. valida Boulay; Schultka, 24-25, Taf. 27, figs 7, 8 (fragmentary specimens which are not wholly characteristic of Alethopteris urophylla, but certainly not Alethopteris valida).

1996 Alethopteris lonchitica (Schlotheim); Brousmiche Delcambre, Mercier \& Coquel, 83: pl. 3, fig. 7 (same specimen as in Brousmiche-Delcambre et al., 1995: photo 6); pl. 4, figs 1, 1a (same specimen as in Brousmiche-Delcambre et al., 1995: photo 5).

p 1996 Alethopteris urophylla (Brongniart) Göppert; Šimůnek, 13-16, pl. X, figs 1, 4 (after Šusta, 1928: Taf. XXXIV, fig. 3), fig. 6 (trichome); pl. XI, fig. 1 (after Purkyňová, 1990: Tab. II, fig. 4), figs 2-7 (cuticles); pl. XII, fig. 1 (? - very fragmentary, difficult to judge), figs 2-4, fig. 5 (?), figs 6-10 (cuticles); pl. XIII, figs 1-8 (cuticles); pl. XIV, fig. 1; text-figs 18-23; non pl. X, figs 2, 3 (comparable with Alethopteris decurrens); non pl. X, fig. 5 (difficult to judge, but possibly Alethopteris havlenae); non text-fig. 17 (possibly Alethopteris havlenae).

1996 Alethopteris lonchitica (Schlotheim) Göppert; Fissunenko in Solovieva et al., 63, 68, 97, pl. 29, fig. 8 .

1997 Alethopteris lonchitica Sternberg; Blake, pl. 2, figs 1-3.

1997 Alethopteris lonchitica Schlotheim; Brousmiche Delcambre et al., 173-174, Pl. II, figs 7-9 (fragmentary).

1998 Alethopteris cf. lonchitica Schlotheim; Brousmiche Delcambre, Mercier \& Coquel, 108, pl. 13, figs 11, 12 (very fragmentary).

Alethopteris valida Boulay; Brousmiche Delcambre, Coquel, Mercier \& Sartori, P1. III, figs 2, 3 (together with Neuralethopteris schlehanii), fig. 4 [with Paripteris gigantea (Sternberg, 1823) Gothan, 1953); non p. 554, P1. III, fig. 1 (=Alethopteris valida].

v 2000 Alethopteris lonchitica; Álvarez-Vázquez, Figs 2, 4 (name only).

v 2001 Alethopteris urophylla Brongniart; Wagner, 63, fig. 60 (same as figured as Alethopteris lonchitica by Álvarez-Vázquez, 1995: lám. 14, figs 2, 2a).

? 2002 Alethopteris urophylla (Brongniart) Presl; Blake et al., 264, 269, 291, 292, pl. XVIII, figs 3, 5.

2002 Alethopteris decurrens (Artis) Zeiller; Blake et al., 264, 268, 291, pl. XVIII, fig. 2.

p 2003 Alethopteris lonchitica (Schlotheim) Sternberg; Josten \& van Amerom, 49, Taf. 92, figs 1, 4, 5; Taf.
95, fig. 8; non Taf. 92, figs 2, 3 (= Neuralethopteris sp. indet.?); non Taf. 93, fig. 1 (included with doubt in Alethopteris lancifolia by Wagner, 2005); non Taf. 93, figs 2, 3 (a single specimen to be compared with Neuralethopteris neuropteroides).

2003 Alethopteris lonchitica; Gil Fernández, 41, Fig. 22. 2004 Alethopteris lonchitica Sternberg; Cleal \& Thomas, Fig. 5c (fragmentary).

\section{Excludenda}

1868 Alethopteris lonchitidis Sternberg var. brevifolia Roehl, 72, Taf. XXI, fig. 9 (Alethopteris lonchitides var. brevifolium in plate explanation) (referred to Alethopteris valida by Kidston, 1886: 133, and by Crookall, 1955: 13, with doubts).

1879-80 Alethopteris lonchitica Schlotheim; Lesquereux, 177179, pl. XXVIII, figs 7, 7a (= Alethopteris lancifolia - although Lesquereux's description of veins forking once or simple does not seem to fit).

1904 Alethopteris lonchitica (Schlotheim); Arber, 305, pl. 20, figs 11, 15 (= Neuralethopteris rectinervis (Kidston, 1888) Laveine, 1967 acc. to Laveine, 1967: 120).

1905 Alethopteris lonchitica Schlotheim; Vinassa de Regny \& Gortani, 483, Tav. XII, figs 10-12 (fragmentary specimens which cannot be judged properly from the photographs published at natural size; but suggestive of Alethopteris lonchitica $=$ Alethopteris ambigua; these specimens from the Stephanian of the Carnic Alps were included, with doubt, in the synonymy of Alethopteris leonensis by Wagner, 1968, a species which, according to Gillespie \& Pfefferkorn, 1986, is synonymous with Alethopteris virginiana).

1908 Alethopteris lonchitica Schlotheim; Renier, 37, fig. $12 \mathrm{~h}$ (drawing of a single pinnule of a specimen figured more completely by Deltenre in Renier et al., 1910: pl. 92, fig. a) (= Alethopteris lancifolia).

1910 Alethopteris lonchitica (Schlotheim); Deltenre in Renier et al., pl. 92, fig. a (part of the specimen refigured by Gómez-Alba, 1988: lám. 6, fig. 3) (= Alethopteris lancifolia).

1913-14 Alethopteris lonchitica Unger (sic); Bureau, 321-322, Pl. LXXIX, fig. 7 (= Neuralethopteris sp. indet.), fig. 8 (= Neuralethopteris?).

1938 Alethopteris lonchitica (Schlotheim); Bell, 67, pl. LXI, fig. 5 (refigured by Zodrow \& Cleal, 1998: pl. 2, fig. 3). Fragment showing incomplete pinnules that may be attributed to the Alethopteris lonchitifolia-westphalensis complex.

1939 Alethopteris lonchitica Schlotheim; Janssen, 143, Fig. 129 (mentioned in Leary, 1976) (difficult to judge from the illustration, but comparable to Alethopteris lonchitifolia).

1939b Alethopteris lonchitica Schlotheim; Jongmans, 31, 37, Taf. VI, fig. 18 (a fragment showing three pinnules which might belong to Alethopteris valida).

1952 Alethopteris lonchitica Brongniart (sic); Jongmans, pl. XXI, fig. 149 (fragmentary specimen, poorly figured; either Neuralethopteris neuropteroides or Alethopteris sp. indet.).

1953b Alethopteris lonchitica Schlotheim; Jongmans, 42, 43, pl. 9, figs 41-45a (probably Neuralethopteris neuro- 
pteroides - Jongmans already expressed some doubt, mentioning a possible confusion with Neuralethopteris schlehanii).

1957 Alethopteris lonchitica Brongniart (sic); Kindelán, 99, fig. 4-4 (fragmentary specimen, difficult to judge, but clearly not Alethopteris urophylla).

1958 Alethopteris lonchitica Schlotheim; Langford, 241, Figs 438, 439 (also mentioned in Leary, 1976) (to be compared with Alethopteris lesquereuxii)

1962 Alethopteris lonchitica (Schlotheim) Gothan; Barthel, 40, Taf. XL, figs 1-3 (= Alethopteris lonchitifolia); Taf. XL, figs 4-7; Taf. XLI, figs 1-4 (epidermal structure).

1962 Alethopteris lonchitica (Schlotheim); Bell, pl. XLII, fig. 4 (= ? Alethopteris cf. davreuxii).

1964 Alethopteris lonchitica (Schlotheim) Unger f. serli (Brongniart) Gothan; Drägert, 41, Taf. 2, fig. 5 (to be compared with Alethopteris havlenae).

1966 Alethopteris lonchitica Zeiller (sic); Migier, 85, 89, Tab. VIII, figs 1-2 (difficult to judge from the illustration; probably indeterminable).

1970 Alethopteris lonchitica (Schlotheim) f. serlii (Brongniart) Gothan; Havlena, pl. I, fig. 13; Pl. II, fig. 5 (only one specimen which is assigned to Alethopteris havlenae by Šimůnek, 1996: 10).

1972a Alethopteris lonchitica Schlotheim; Biţoianu, pl. II, fig. 13 (= too fragmentary and poorly figured, but possibly a Neuralethopteris).

1972b Alethopteris lonchitica Schlotheim; Biţoianu, pl. IV, fig. 3 (= too fragmentary, possibly Neuralethopteris).

1973 Alethopteris lonchitica Zeiller (sic); Biţoianu, pl. III, fig. 29 (to be compared with Alethopteris westphalensis).

1973 Alethopteris lonchitica Schlotheim; Caride, Greber \& Ortuño, pl. II, figs 1, 1a; pl. III, figs 3, 3a [to be compared with Alethopteris westphalensis and Alethopteris missouriensis, and probably the same species as that recorded (erroneously) as Alethopteris decurrens by Caride et al., 1973: pl. II, figs 2-3].

1977 Alethopteris lonchitica (Schlotheim) Sternberg; Leary \& Pfefferkorn, 25-27, text-fig. 9A-D, pl. 9, figs 1-6 (specimens previously mentioned in Leary, 1976: 4) (to be compared with Alethopteris havlenae).

1978 Alethopteris lonchitica; Gillespie, Clendening \& Pfefferkorn, 100, pl. 35, fig. 3 (nervation diagram); pl. 36, fig. 7 (pointed pinnules tending to a lanceolate shape not suggestive of Alethopteris urophylla).

1979 Alethopteris lonchitica (Schlotheim) Göppert; Anisimova, Tab. XXXIV, fig. 4 (= Alethopteris decurrens).

1982 Alethopteris lonchitica (Schlotheim) Brongniart; Oleksyshyn, 98-99, figs 19F-H (to be compared with Alethopteris missouriensis).

1985 Alethopteris cf. lonchitica (Schlotheim) Brongniart; Lyons et al., pl. XI, fig. b (very fragmentary; either Neuralethopteris sp. indet. or Alethopteris sp. indet.).

1986 Alethopteris lonchitica (Schlotheim); Purkyňová, Tab. IV, fig. 3 (= Alethopteris havlenae) (Šimůnek, 1996: 14 , includes this specimen in Alethopteris urophylla).

2003 Alethopteris cf. lonchitica; Laveine et al., 506-507, pl. X, figs 1-2 (probably to be compared with Neuropteris obliqua sensu lato).
? 2007 Alethopteris lonchitica; Opluštil et al., pl. VIII, fig. 4 [figured at x 0.2 , which does not allow the identification to be substantiated. N.B. there are no previous records of Alethopteris lonchitica (auctorum) = Alethopteris urophylla from the Radnice Formation in Central Bohemia. It may be assumed that the authors identified Alethopteris lonchitica with Alethopteris lonchitifolia following Zodrow \& Cleal's, 1998, misidentification; Nĕmejc, 1936, did not record Alethopteris lonchitica from the Radnice measures, but figured specimens of the Alethopteris lonchitifolia-missouriensis-westphalensis complex].

2007 Alethopteris lonchitica Sternberg; Šimůnek, 394, pl. 2, figs 6-8 (cuticles); pl. 5, fig. 2 (= Alethopteris lonchitifolia).

Diagnosis (after Brongniart, 1834): "P. foliis tripinnatifidis; pinnis primariis apice tantùm pinnatifidis, pinnulis linearibus longissimis decurrentibus; inferiùs bipinnatifidis pinnis elongatis, in pinnulam linearem maximam desinentibus; pinnulis lateralibus sub conformibus, in pinnis inferioribus majoribus, obliquis, oblongis, obtusiusculis, sub contiguis, basi paululùm dilatatis, decurrentibus et connatis, integerrimis, lineâ impressâ marginatis; nervio medio angusto apprimè notato, nervulis tenuissimis simplicibus vel plerumque furcatis vix obliquis".

This translates as follows: Tripinnate frond; terminal of primary pinnae clearly pinnatifid, pinnules long, linear and decurrent: lower bipinnate pinnae elongate, with linear pinnules developed to maximum extent; lateral pinnules subparallel in most of lower pinnae, oblique, oblong, blunt, hardly contiguous, with the base only a little expanded, decurrent and connate, entire, margin distinct; midvein thin but well marked, nervules thin, simple or several times forked, hardly if at all oblique.

Holotype: Tripinnate frond fragment from Merthyr Tydfill, South Wales. British Geological Survey collection Cat. $\mathrm{n}^{\circ}$ 5127A, as figured photographically in the present paper (Figs 2-4).

Redescription of holotype: A tripinnate fragment of a frond showing near-terminal parts of two pinnae of the penultimate order with elongate pinnules, passing rapidly into pinnae of the last order with strongly vaulted pinnules (i.e. showing a convex limb) which display a compression border. Rachises are straight, only 1-2 mm wide (as corresponds to near-terminal parts of major pinnae). Pinnules attached obliquely, at c. $60-80^{\circ}$ angle, very narrowly confluent, slightly asymmetrical, with a sloping base and a marked constriction on the acroscopic side. Last order pinna terminals elongate, parallel-sided but tapering in the upper part, as are the elongate pinnules above the passage to pinnae of the last order. Pinnule length in the terminals diminishes upwards, becoming only slightly longer than the lateral pinnules. Apical pinnules always well individualised. Lateral pinnules parallel-sided to slightly biconvex, with bluntly acuminate apices. Length-breadth ratios 2-3 for standard lateral pinnules, with higher ratios for more elongate pinnules. Pinnule length variable as against more constant width, which varies between 
3 and $4 \mathrm{~mm}$. Midrib straight, well marked, deeply immersed in thick pinnule limb, practically non-decurrent and extending to near the pinnule apex. Lateral veins thin, numerous (difficult to count exactly), fairly regularly disposed, generally once forked, slightly curved near the midrib, relatively straight and reaching the pinnule margin at right angles.

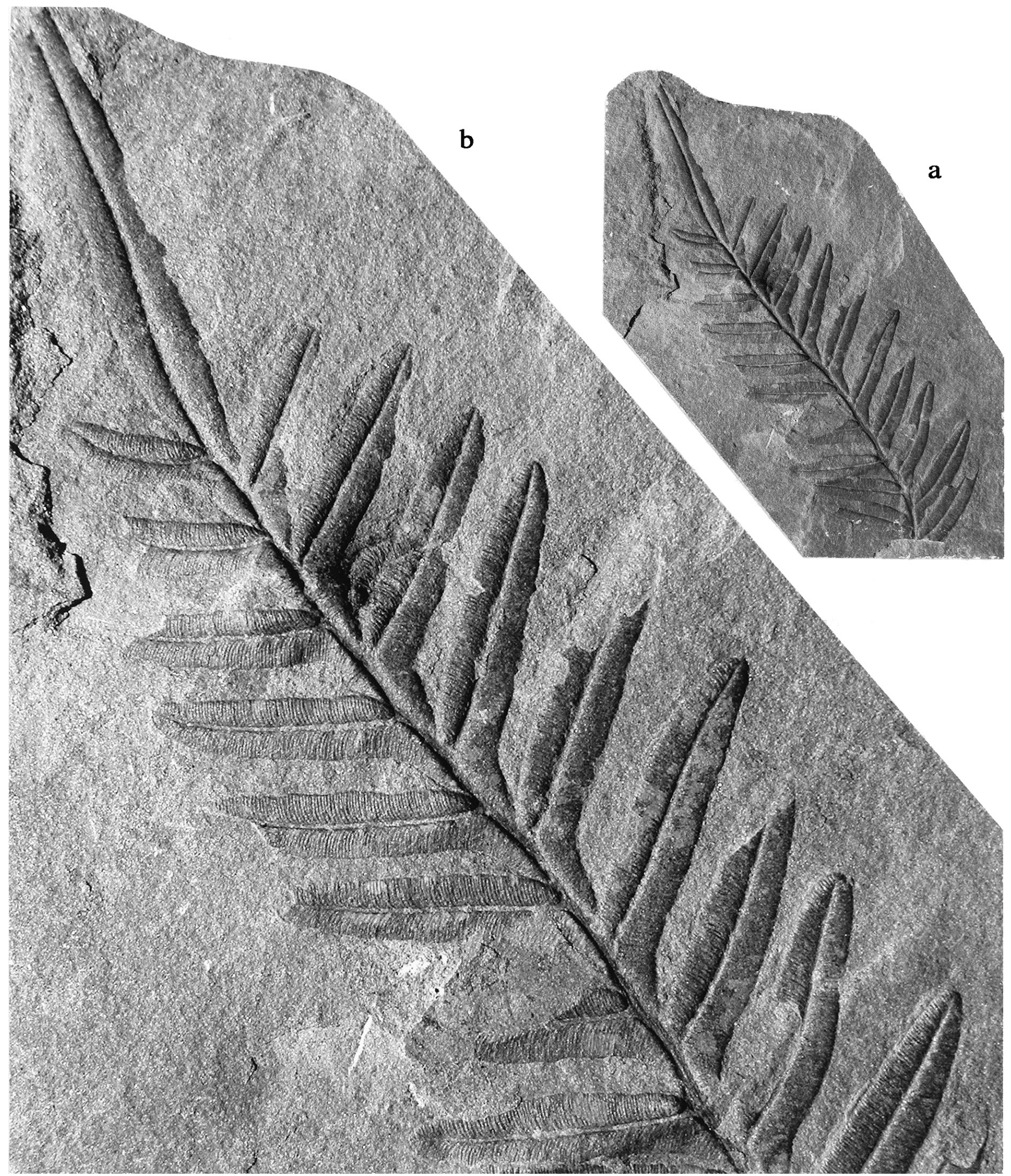

Figure 6. Terminal part of a last order pinnae with elongate, narrowly confluent pinnules, with bluntly acuminate apices. Note the elongate terminal. Origin: Rig Burn, about 2 km southwest of Kirkconnel, Sanquhar Outlier, Southern Uplands, Scotland, lower Duckmantian (loc. 9716). (a) x 1; (b) x 3. Collected by K. Higgs, 1971. Repository: Centro Paleobotánico, Córdoba. 


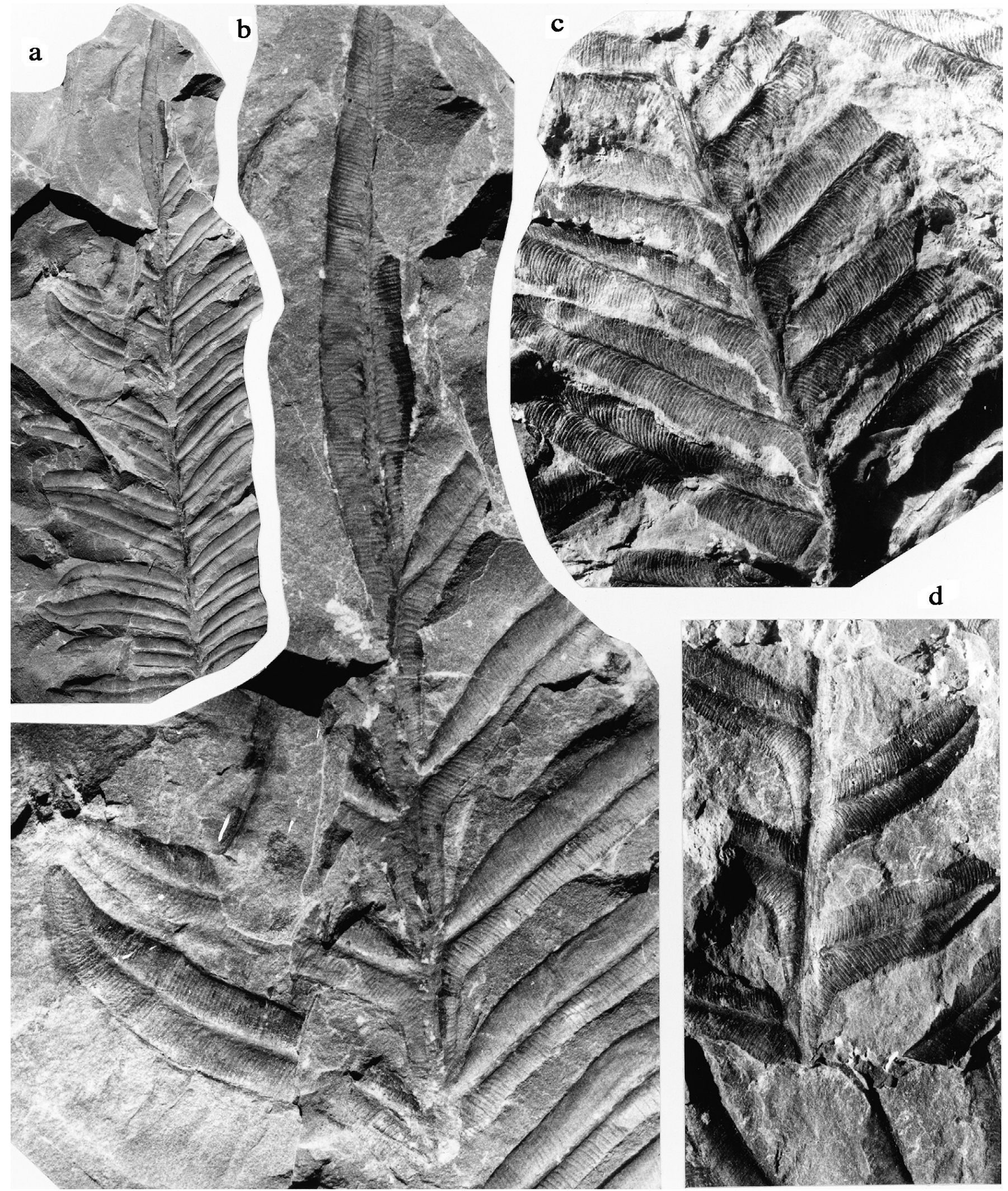

Figure 7. a, Pinna of the penultimate order with elongate pinnules and ditto terminal (x 1). b, Enlargement (x 3 ) of part of the same specimen. c, Relatively large pinnules (x 3). d, Smaller, more average size pinnules (x 3). All specimens from GSC loc. 205 (coll. H.M. Ami, 1899), Springhill Mines, Nova Scotia, Canada, Langsettian. Repository: Geological Survey of Canada, Ottawa. 


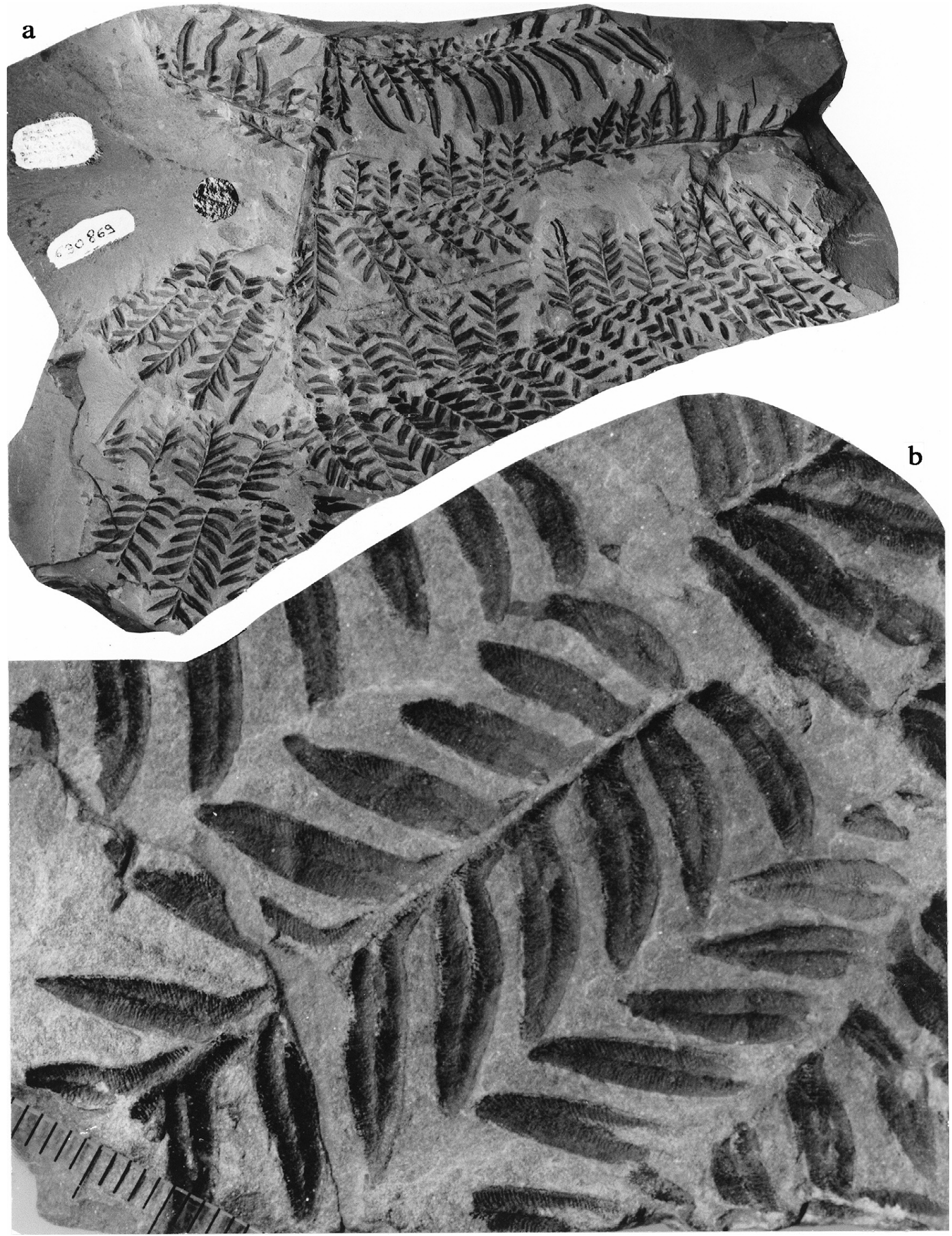

Figure 8. a, A large frond fragment representing a medial portion transitional to the terminal part of an antepenultimate pinna, at half the natural size (x 0.5). It shows the transition between last order pinnae and pinnules, displaying the apical growth which leads to elongate shapes of apical as well as the lateral pinnules. Specimen figured previously by Buisine, 1961: pl. XIII, fig. 1. Origin: Nord/Pas-de-Calais Coalfield, Aniche, Bernicourt, Cécile Seam, Langsettian. Repository and photograph: Musée de Géologie, Ville de Lille. b, Part of the same specimen (x 3) showing average-sized lateral pinnules with characteristic constriction on the acroscopic side and a narrow limb connecting pinnule bases. The enlargement depicts pinnae in the lower left hand corner of the large specimen of Fig. 8a. Photo: P. De Bleeckere, Musée de Géologie, Ville de Lille. 


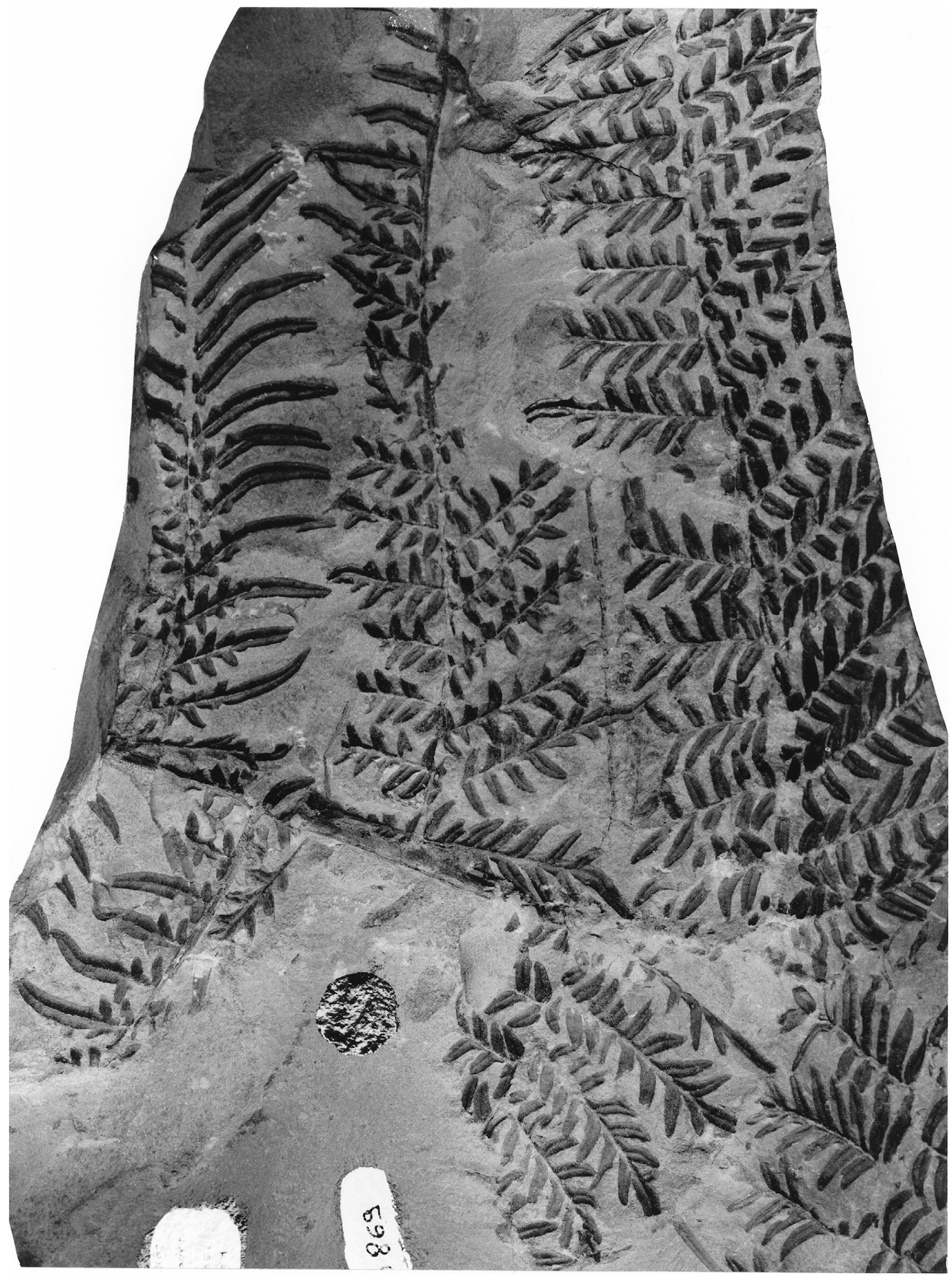


General description of species (based on the sum total of specimens admitted from the different parts of the world - compare list of synonymy): Large fronds (at least tripinnate) with relatively thin rachises and last order pinnae touching laterally. Pinna terminals elongate, parallel-sided, but tapering in the upper part, and similar to the more elongate pinnules. Lateral pinnules oblique, well separated, decurrent, with narrowly confluent bases (which do not normally constitute a common limb alongside the rachis), parallel-sided but tapering in the upper part, with a bluntly acuminate apex. Pinnule lamina strongly convex ("vaulted"), with thin, but distinct midrib immersed in the lamina. Pinnule length extremely variable, depending on the position in major pinnae, with an abrupt transition between last order pinnae and markedly elongate pinnules in the top part of pinnae of the penultimate order. Pinnule width relatively constant even where pinnule length increases markedly (apical growth). Midrib straight and extending into apical part of pinnules. Lateral veins thin, perpendicular to both midrib and pinnule margin, curving only slightly at the point of departure from the midrib; they are generally once forked, more rarely with a second bifurcation and very rarely simple. Vein density 48-55 veins per $\mathrm{cm}$. Length/breadth ratio extremely variable. Dimensions: 9-45 mm length at 3-5 width.

Comparisons: Alethopteris decurrens (Artis, 1825) Frech, 1880 has narrower, broadly confluent and, generally, more widely spaced pinnules which tend to be slender and more parallel-sided. The vein density is variable in relation to the pinnule width, but seems to be generally 30-40 veins/ cm (N.B. although Buisine, 1961, mentions a vein density of 40-50 veins/cm, he also characterises the venation as "peu serrée" which is contradictory). The wider spaced veins of Alethopteris decurrens pinnules seem more irregular than those of Alethopteris urophylla. However, certain parts of Alethopteris urophylla, which are characterised by more elongate pinnules, may resemble Alethopteris decurrens (hence the doubts attached to the drawing of Alethopteris heterophylla). Kidston (1886: 134) observed gradual transitions between Alethopteris decurrens and Alethopteris lonchitica (auctorum), and Crookall (1955) noted their similar stratigraphic range. The species introduced by Stockmans \& Willière (1952-53) as Alethopteris edwardsii (1953: 240; 1952: pl. LVI, figs 9, 9a) and Alethopteris tectensis (1953: 241; 1952: pl. LVI, figs 8, 8a) from one and the same locality in the Assise d'Andenne, upper Namurian (Yeadonian) of Belgium, and which the present writers regard as probably belonging to one and the same species, are comparable to Alethopteris decurrens in pinnule shape and size, but apparently possess a wider nervation. Additional specimens of Stockmans \& Willière's species, as figured by Josten (1983) from the middle Namurian of the Ruhr District, western Germany, reinforce this impression.
Alethopteris lancifolia Wagner is characterised by more tapering, lanceolate pinnules of generally larger size and a basal width which is, on the whole, larger than that of Alethopteris urophylla. Vein density of $45-50$ veins/cm is similar to that of Alethopteris urophylla. It may be that Alethopteris lancifolia should be regarded as an extreme form of Alethopteris urophylla, but this can only be demonstrated in very large frond fragments which are not available at present. The most complete specimens known at present (e.g. that figured here as Figs 8-10) do not show complete morphological overlap. Its stratigraphic occurrence is the same as that for Alethopteris urophylla. N.B. Two of the specimens figured as co-types of Alethopteris lancifolia by Wagner (1961: pl. 2, figs 6-7) should be excluded because of pinnule shape, the much wider confluence between pinnules, and a wider venation. A comparison with Alethopteris valida is suggested. No revision of Alethopteris lancifolia is attempted here, but compare Wagner (2005).

Alethopteris corsinii Buisine (1961: 115-125, pls XXIXXVI) shows pinnules similar in shape and size to those of Alethopteris urophylla. However, pinnule insertion seems on the whole more perpendicular and the constriction on the acroscopic side is consequently less apparent. Its nervation consists of a well marked midrib and perpendicular lateral veins showing a mixture of simple veins and those forked at the midrib. According to Buisine (1961: 116), the vein density is c. 30 veins per cm, which is less than that admitted for Alethopteris urophylla.

Alethopteris hermetetii Buisine (1961: 179-184, pl. XLIX) is rather similar to Alethopteris corsinii. Indeed the pinnules are nearly perpendicular, and therefore lack the more markedly asymmetrical aspect of Alethopteris urophylla pinnules. Vein density is given as c. 30 veins per $\mathrm{cm}$ according to Buisine, which is the same as that recorded for Alethopteris corsinii.

Alethopteris lonchitica Schlotheim ex Sternberg has pinnules of similar size, but, on the whole, a little more slender, parallel-sided, with a more broadly rounded apex, and showing a wide midrib extending to the tip of the pinnule. Its lateral veins are more widely spaced. The pinnules of Alethopteris lonchitica tend to be more pecopteroid, and more nearly perpendicular to the rachis. Alethopteris friedelii (= Alethopteris ambigua) is a synonym of Alethopteris lonchitica. This is an upper Westphalian species which ranges into basal Stephanian.

The Bolsovian species Alethopteris bertrandii Bouroz, 1956 possesses stiff, almost parallel-sided pinnules, tapering in the upper part, with a pointed apex. The very large, elongate terminals are regarded as characteristic. Its venation is characterised by a distinct, rather wide midrib and

Figure 9. Most of the specimen figured as figure 8a, at natural size (x 1). This shows quite well the transitions between lengthened pinnules in the upper parts of two penultimate pinnae and small pinnae in which the pinnules lengthen by apical growth. Photo: P. De Bleeckere, Musée de Géologie, Ville de Lille. 
fairly regularly disposed lateral veins which are generally once forked. Vein density is given by Bouroz (1956: 141) as c. $24-28$ veins per $\mathrm{cm}$. The similar species Alethopteris jankii Coquel \& Laveine (Coquel \& Laveine, 1979) possesses even larger pinnules, which are marginally wider and therefore apparently not quite as pointed as in Alethopteris bertrandii. However, these differences as observed by Coquel \& Laveine (1979) are only minor, and the question may be raised whether these two species should not be regarded as one and the same. Coquel \& Laveine (1979) also observed the close resemblance which exists with regard to Taeniopteris? missouriensis (White, 1899: 140-144, pl. XL, figs 1-7) which is clearly an Alethopteris, and which might well prove to be the same as Alethopteris bertrandii. Even the vein density is exactly the same (2428 veins per $\mathrm{cm}$ as mentioned by White, 1899: 141). If White's species is recognised as an Alethopteris, its specific name coincides with that of Alethopteris serlii var. missouriensis White (which was elevated to species rank by Wagner, 1968), thus producing a nomenclatorial clash. However, since Alethopteris serlii var. missouriensis is described on page 118 of White's Memoir, and Taeniopteris? missouriensis on page 140 of the same memoir, the former would have priority. It is also noted that White's illlustrations of Alethopteris (ex Taeniopteris?) missouriensis are drawings, which need to be checked on accuracy. In view of the homonymy, it is just as well that Alethopteris bertrandii exists.

Alethopteris densinervosa Wagner, 1968, an upper Westphalian species from northern France (Bolsovian, lower Asturian), shows more biconvex pinnules which tend to be more bluntly acuminate than those of Alethopteris urophylla and which are more widely confluent. It shows generally larger pinnules and thus resembles more closely Alethopteris lancifolia (see comments in Wagner, 1968: $62)$. Vein density is quite similar in all three species.

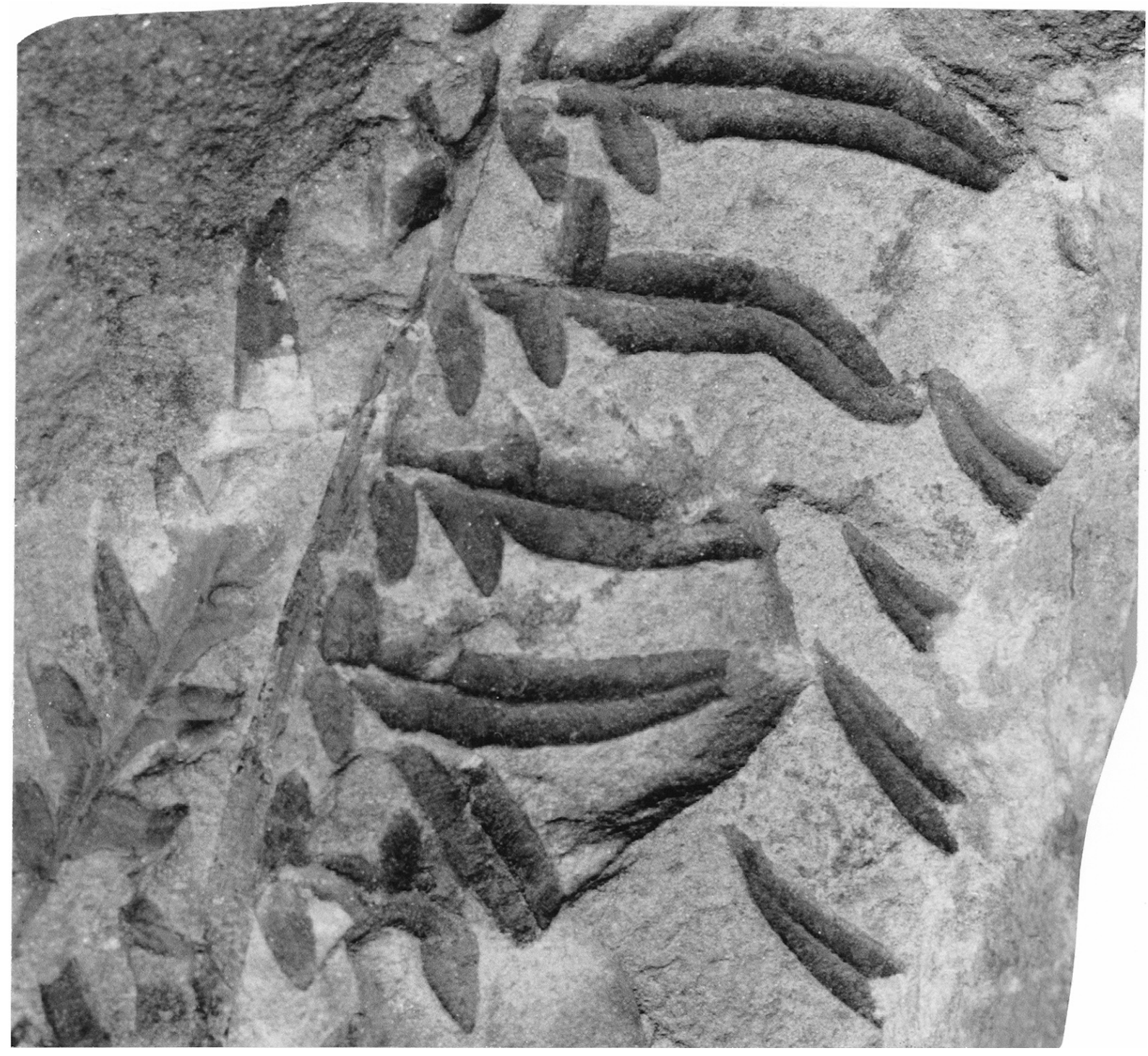

Figure 10. Small fragment of Figs 8a and 9 (x 3). Photo: P. De Bleeckere, Musée de Géologie, Ville de Lille. 
Alethopteris westphalensis Wagner, 1968 also shows parallel-sided pinnules which are, on the whole, more broadly confluent than those of Alethopteris urophylla, and which tend to be subperpendicular to the rachis. Its pinnules are less asymmetrical than those of Alethopteris urophylla. Vein density is $30-35$ veins per $\mathrm{cm}$ (Wagner, 1968: 154). This species is fairly similar in pinnule shape and insertion, as well as the vein density, to Alethopteris corsinii, but shows only occasional simple veins whereas these are recorded as common for Alethopteris corsinii.

Alethopteris brevis Weiss, 1869 (Weiss, 1869-72: 8283 , Taf. XI, fig. 1) is a lower Rotliegend species characterised by narrowly confluent, tapering pinnules with rounded apices and a vaulted limb. Their insertion is subperpendicular to slightly oblique. Although comparable, they are, on the whole, more widely spaced than those of Alethopteris urophylla. The terminals of Alethopteris brevis are characterised by a small apical pinnule, whereas these are elongate in Alethopteris urophylla. Its nervation shows a rather strong midrib (apparently wider than that of Alethopteris urophylla pinnules) and at least once forked, perpendicular lateral veins which are rather widely spaced (c. 30 veins per $\mathrm{cm}$ ). The above description is based on lower Permian material from Valdeviar in SW Spain (Wagner \& Mayoral, 2007). The original description and illustration by Weiss (1869-72) relates to a single specimen from the lower part of the frond and which shows more pecopteroid, perpendicularly inserted pinnules. Pinna fragments of these characteristics also occur in the Valdeviar locality, which has yielded about a dozen remains showing the range in morphological variation which is not apparent from Weiss's illustration.

\section{TAXONOMIC DISCUSSION}

There can be no doubt that Alethopteris urophylla, as figured and described by Brongniart (1834), is the same as most specimens recorded as Alethopteris lonchitica in the literature, i.e. Alethopteris lonchitica (auctorum). In fact, Brongniart's species is almost invariably placed in synonymy with Alethopteris lonchitica as understood by the different authors, i.e. independent from the type of Alethopteris lonchitica which the present writers regard as identical to Alethopteris friedelii (=Alethopteris ambigua). This species is quite different to Alethopteris lonchitica (auctorum). A question mark is raised with regard to Alethopteris lonchitica as figured by Brongniart (1833). The specimens illustrated by Brongniart (1833: pls 84,128) are apparently from three different localities, viz. Dudweiler near Saarbrücken, the vicinity of Namur in Belgium, and Newcastle-on-Tyne in northeast England. Brongniart (1833: 275) mentions Schlotheim's type as coming from Silesia, which is in error because the type specimen of Alethopteris lonchitica originated from Saarland in western Germany. It is possible that at least two of the specimens illustrated by Brongniart, i.e. his pl. 84, figs 2 and 7 , which show constricted bases to the pinnules in the lower part of the pinnae, should be assigned to Neuralethopteris neuropteroides (Šusta, 1927) Josten, 1983. Additional remains (pl. 84 , figs $4,5,6$ ) might well belong to the same species, thus leaving only Brongniart's pl. 84, figs 1, 1a as Alethopteris lonchitica (auctorum). It is not immediately obvious how Brongniart distinguished this specimen from Alethopteris urophylla which appears in the comparisons as possessing the same kind of passage from elongate pinnules to pinnae of the last order in the terminal parts of higher order pinnae. Of course, this rapid passage from pinnules to pinnae is not uncommon in Alethopteris in general, although Brongniart's Alethopteris lonchitica of his plate 84, figs 1, 1a, and the holotype of Alethopteris urophylla do share this character to a marked degree. Brongniart was most interested in comparing with the morphological characters of certain Recent ferns, and referred more particularly to Pteris. After diagnosing "Pecopteris" urophylla (Brongniart, 1834: 290), he mentioned that this species also belonged to the Pteris group, but emphasised what he regarded as distinct traces of marginal fructifications, referring undoubtedly to a compression border as occurs in thicklimbed pinnules of Alethopteris. This is clearly apparent in the holotype of Alethopteris urophylla (see Figs 3, 4). Discounting this preservational character, one may well pose the question whether Brongniart's pl. 84, figs 1, 1a, attributed to Alethopteris lonchitica is not the same species as Alethopteris urophylla. There is no apparent difference in the shape and size of pinnules, and the elongate terminals which are partially visible in Brongniart's pl. 84, fig. 1 , are also identical to those of Alethopteris urophylla. The only possible difference is in the venation which appears a little more widely spaced in Brongniart's Alethopteris lonchitica (pl. 84, fig. 1a), i.e. c. 30 veins/cm. Alethopteris urophylla has a higher vein density of c. 48-55 veins per $\mathrm{cm}$ on the pinnule margin. Brongniart's drawing shows both once forked and simple veins, which accords rather well with his statement (Brongniart, 1834: 290) of both simple and bifurcate (and even more than once bifurcate) veins in Alethopteris urophylla. Brongniart's pl. 84, figs 1, 1a (for Alethopteris lonchitica) is here included tentatively in the synonymy of Alethopteris urophylla which seems to have been mainly distinguished on the presence of a compression border, misinterpreted as marginal fructifications. Brongniart's pl. 128 shows a possibly different species which Buisine (1961) identified with Alethopteris friedelii. This is the species regarded here as identical with Alethopteris lonchitica.

Alethopteris lonchitica (auctorum) as figured from the lower Westphalian of Europe, seems to have been inspired mainly on the well preserved specimen from the North of France illustrated by Zeiller (1886: pl. XXXI, figs 1, 1a), although usage has been variable. Zeiller's specimen is 
broadly similar to Alethopteris urophylla, although there is a suggestion of more biconvex, not quite as parallelsided pinnules, as occur in the type specimen of Aletho- pteris urophylla. Zeiller's nervation diagram shows predominantly once to more than once bifurcate nervules with only occasional simple veins. The vein density appears to
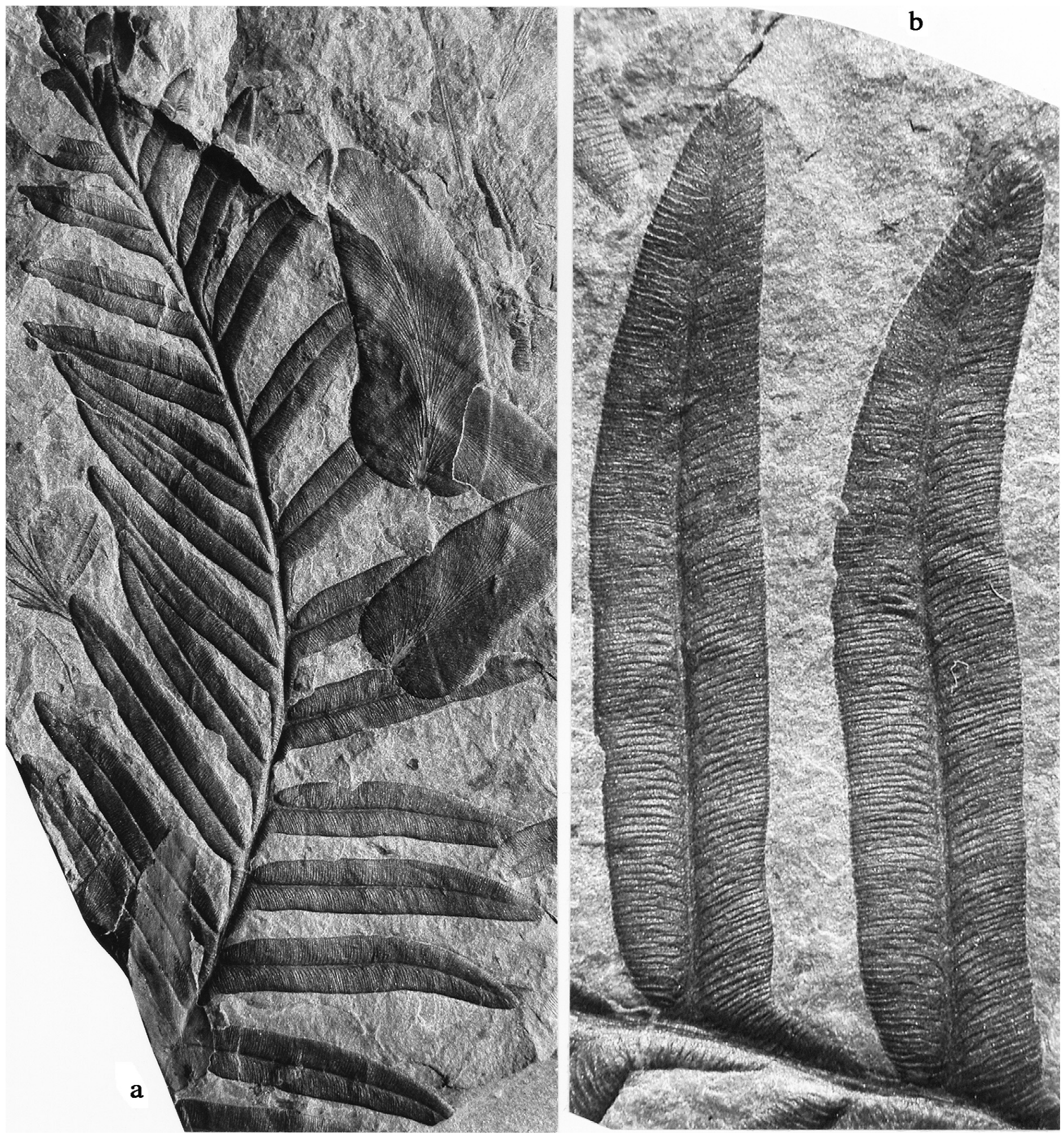

Figure 11. a, Last order pinna (x 2), with elongate, narrowly confluent pinnules; pinna terminal incomplete. Detached pinnules of Paripteris gigantea associated. b. Two pinnules of the same specimen, enlarged (x 6) to show the once to twice bifurcate veins. Origin: Cabeza de Vaca opencast site near Belmez, Peñarroya-Belmez-Espiel Coalfield, province Córdoba, Spain, upper Duckmantian (loc. CV-004). Repository: Centro Paleobotánico, Córdoba. 
be around 46 veins/cm which accords with that found in Alethopteris urophylla. The exhaustive documentation published by Buisine (1961) from the same area of northern France insists on the presence of once and twice bifurcate nervules, with a minimum of 45 veins per $\mathrm{cm}$ on the pinnule margin, and an average of about 50 veins $/ \mathrm{cm}$. Buisine (1961: 109) seems to admit most of Brongniart's figures attributed to Alethopteris lonchitica as belonging to the species which Zeiller (1886-88) described from northern France, but he excluded the specimen figured in the lower part of Brongniart's (1833) pl. 128, which he compared with Alethopteris friedelii, probably correctly. This, ironically, would be the real Alethopteris lonchitica. Although Brongniart's illustration on pl. 128 leaves room for doubt, one would agree with Buisine that this specimen is not the same as those customarily regarded as Alethopteris lonchitica (auctorum) (= Alethopteris urophylla).

Buisine (1961) also discussed the species Alethopteris discrepans Dawson $(1862,1868)$ as figured more reliably by Stopes (1914), and, in agreement with Stopes, included this species in the synonymy of Alethopteris lonchitica (auctorum). Stopes illustrated material from Dawson's original locality, the "Fern Ledges" at Saint John, New Brunswick (Canada), as well as even more fragmentary remains from the Joggins section on the Bay of Fundy, Nova Scotia. One of the larger fragments of this species figured originally as a diagrammatic drawing by Dawson (1871) was refigured photographically by Stopes (1914: pl. XIII, fig. 31), who added a further, previously unfigured specimen from the Dawson Collection (op. cit.: pl. XII, fig. 30). Although the venation is unclear, these remains could well belong to Alethopteris urophylla. She also figured some additional, very fragmentary remains of Alethopteris discrepans from the Joggins section in Nova Scotia (Stopes, 1914: pl. XIII, figs 32,33$)$. The venation of these specimens is unclear. Better preserved, more complete specimens from the Joggins section were collected more recently by Donald Reid, and made available for description by the present writers. These specimens are similar to Alethopteris urophylla, but show relatively broader pinnules which are more broadly confluent and which show a wider nervation. Perhaps, they are to be attributed to Alethopteris corsinii. The poorly preserved fragments figured by Stopes might well belong to the same species, but this can only be conjectural. On the other hand, the well preserved specimens figured by Bell (1966) from the Springhill Mines, in the same general area of Nova Scotia, under the name of Alethopteris lonchiti$c a$, can undoubtedly be assigned to Alethopteris urophylla (see also Figs 7a-c in the present paper). A reexamination of various other specimens from the Springhill Mines allows the observation that predominantly once bifurcate veins exist, with an admixture of simple veins, and a vein density of around $25-35$ veins $/ \mathrm{cm}$. This is markedly lower than that of the material from northern France, and also the type of Alethopteris urophylla.
The Alethopteris serlii as figured and described by Buisine (1961) from the North of France is not Brongniart's species as Wagner (1968) has pointed out. Zodrow \& Cleal (1998: 79) have recently confirmed that Alethopteris serlii has been widely misidentified in the literature. They also mentioned that many authors (e.g. Gothan, 1953) regarded Alethopteris serlii as merely a growth form of Alethopteris lonchitica, meaning Alethopteris lonchitica (auctorum). Part of Buisine's Alethopteris serlii has been redescribed as Alethopteris densinervosa by Wagner (1968). This is a species which Zodrow \& Cleal (1998) incorporate with their Alethopteris lonchitica (which they identified mistakenly with Alethopteris lonchitifolia). Alethopteris densinervosa and Alethopteris lonchitifolia are closely similar, and have been regarded as possible regional varieties by Wagner (1968). This concept is not discussed by Zodrow \& Cleal (1998), who based the intraspecific variation of their species on the analysis made by Scheihing \& Pfefferkorn (1980) on the Alethopteris remains from a single locality in Pennsylvania. They agree with Scheihing and Pfefferkorn that Alethopteris densinervosa, Alethopteris lonchitifolia, Alethopteris missouriensis, and Alethopteris westphalensis are all to be regarded as belonging to a single taxon (N.B. Scheihing and Pfefferkorn also included Alethopteris serlii, which is quite different, as Zodrow and Cleal rightly observed). It is noted here that different species may have partly overlapping characters, a fact that is not taken into account by either Scheihing \& Pfefferkorn (1980) or Zodrow \& Cleal (1998). Their single (composite?) species was identified with Alethopteris lonchitica by Zodrow \& Cleal (1998), an identification which is rejected by the present writers. Recognition of a single species as meant by Zodrow and Cleal would imply acceptance of White's Alethopteris serlii var. missouriensis as the taxon enjoying priority in the Alethopteris missouriensis-A. lonchitifolia-A. westphalensis-A. densinervosa complex. In this case, all the specimens figured and described by Buisine (1961) as Alethopteris serlii would have to be assigned to Alethopteris missouriensis. However, this is not the opinion of the present writers, who prefer to distinguish two different taxa in Buisine's material, viz. Alethopteris densinervosa and Alethopteris westphalensis. The beautiful set of plates depicting large and well preserved remains of Alethopteris serlii sensu Buisine (non Brongniart) from the lower part of the Assise de Bruay (Bolsovian) of northern France, probably represents two different species. One of these is characterised by biconvex to more parallel-sided pinnules (in relation to the length of pinnules) and a rather dense venation. This is the taxon described as Alethopteris densinervosa (Wagner, 1968: 59), with a vein density of 40-45 per $\mathrm{cm}$. The other species is characterised by more slender pinnules with a slightly wider nervation and, above all, no apparent constriction on the acroscopic side. This is identified with Alethopteris westphalensis. Wagner (1968: 59) singled out a specimen 
figured by Buisine (1961) on his pl. VIII, figs 2, 2a, for a tentative assignment to Alethopteris urophylla. This opinion is not maintained by the present writers, who prefer to assign this specimen to Alethopteris westphalensis. The specimen in question shows pinnules which are less constricted on the acroscopic side than is customary for Alethopteris urophylla.

Buisine (1961: 89) compared with Alethopteris lonchitica (auctorum) in the sense of Zeiller (1886-88), separating his Alethopteris serlii (i.e. Alethopteris densinervosa and Alethopteris westphalensis) from Alethopteris lonchitica (auctorum) (i.e. Alethopteris urophylla), as occurring in northern France, primarily on stratigraphic criteria. However, Buisine also drew attention to the more asymmetrical aspect of the pinnules of Alethopteris lonchitica (sensu Zeiller), showing a marked constriction of the basal part on the acroscopic side. The pinnules of the lower Westphalian species also appeared to be more narrowly confluent than those of the upper Westphalian taxon from northern France (op. cit.).

Crookall (1955) provided an exhaustive account of Alethopteris lonchitica (auctorum), with respect to localities in the British Isles. He included in the synonymy both Alethopteris urophylla and Alethopteris discrepans. Crookall illustrated two specimens from the Barnsley Seam (lower Duckmantian) in Yorkshire (op. cit.: pl. V, fig. 2; pl. X, fig. 1), and a Scottish specimen of apparently the same Duckmantian age (pl. X, fig. 3). All seem to accord well with Alethopteris urophylla as regards pinnule size and shape. The approximate vein density, as measured on the enlargement of Crookall's pl. X, fig. 3, is around 48 veins $/ \mathrm{cm}$. This agrees with the vein density of Alethopteris urophylla. An additional specimen from the Duckmantian of Yorkshire (Crookall, 1955: pl. V, fig. 1) appears to fall outside the range of variation, and has been attributed to Alethopteris lancifolia by Wagner $(1961,1968)$. Crookall did not express the vein density numerically, but referred to the veins as being numerous. Material from the Barnsley Seam is also figured in the present paper (Fig. 5).

Josten (1991), in his comprehensive account of Westphalian "coal measure" floras of the Ruhr District in western Germany, figured only two specimens of what he called Alethopteris lonchitica. Both are fairly typical of Alethopteris urophylla in general morphology, and vein density (45-55 veins $/ \mathrm{cm})$. These specimens came from Langsettian and Duckmantian strata.

Gothan (1953) recorded Alethopteris lonchitica from the same West German area as dealt with by Josten (1991). He illustrated several different forms; a number of specimens (Gothan, 1953: Taf. 4, figs 2, 3, 5; Taf. 5, figs 1, 4, 5; Taf. 6, figs 2-4) conform to Alethopteris urophylla (= Alethopteris lonchitica sensu Zeiller, as figured later by Josten, 1991), whereas another (Taf. 4, fig. 1) has been ascribed by Wagner (1961) to his Alethopteris lancifolia. Two additional remains, from the middle Namurian (Taf.
4, fig. 3) and the Bolsovian (Taf. 4, fig. 4), respectively, are tentatively attributed to Alethopteris lancifolia and Alethopteris westphalensis. Gothan (1953: Taf. 5, fig. 3) also figured as Alethopteris lonchitica (forma serlii) a specimen which is very different, and comparable to Alethopteris grandinioides Kessler according to Wagner, 1968 (referred to Alethopteris pseudograndinioides by Zodrow \& Cleal, 1998). A specimen figured by Franke (1912) as Alethopteris serli forma platyrachis (only a drawing), was illustrated photographically by Gothan (1953: Taf. 6, fig. 1), as Alethopteris lonchitica forma serli. This specimen was attributed, with doubt, to Alethopteris westphalensis by Wagner (1968), who commented on this specimen in detail. It may be more properly ascribed to Alethopteris havlenae.

Alethopteris vulgatior Sternberg, 1825, which is commonly attributed to Alethopteris lonchitica (auctorum), is based on a holotype of unknown origin. This type specimen has been refigured photographically by Kvaček \& Štraková (1997: pl. 56, fig. 2) at less than natural size (x $0.75)$. The slender, parallel-sided pinnules allow a comparison with Neuralethopteris neuropteroides, rather than Alethopteris lonchitica (auctorum). It is here rejected as a possible synonym of Alethopteris urophylla. Alethopteris vulgatior was renamed Alethopteris sternbergii by Göppert (1836: 295), an illegitimate name as Kvaček \& Štraková (1997) have pointed out. It is noted that Göppert (loc. cit.) compared with the specimens which Brongniart (1833: pl. 84, figs 5-7) figured as Alethopteris lonchitica (pars); these specimens are attributed by the present writers to Neuralethopteris neuropteroides. Ettingshausen (1854) figured as Alethopteris sternbergii a specimen which Wagner (1968) referred to Alethopteris missouriensis. This species is quite different from Alethopteris vulgatior (= Neuralethopteris neuropteroides?).

Although not directly relevant to the synonymy of Alethopteris urophylla, the species "Neuropteris" distans Sternberg, 1825, nomen nudum, but figured as Alethopteris lonchitica by Kvaček \& Kvaček (1992), and Kvaček \& Straková (1997), might belong to either Alethopteris lonchitifolia or Alethopteris westphalensis. Sternberg's type (first figured by Kvaček \& Kvaček, 1992) is stated to originate from Eschweiler near Saarbrücken (Kvaček \& Štraková, 1997: 65).

\section{COMMENTS REGARDING THE FIGURED SPECIMENS}

Brongniart's type specimen from South Wales was figured originally as a lithograph (fide Cleal et al., 2005: 53). A copy of Brongniart's plate is reproduced here as Fig. 1 . It has been taken from the facsimile edition of 1915 (Ed. W. Junk, Berlin). When compared with the photograph of the type specimen in the British Geological Survey Col- 
lection (Figs 2-4), it becomes apparent that Brongniart's artist took certain liberties. The outline of the rock sample was made to look more pretty and, more seriously, the two pinnae were depicted as lying in parallel position, whereas, in fact, they were not exactly. Also, the smaller pinnae were not figured exactly as they were, and this means that certain pinnules were partially restored, as a result of which these pinnules were to some extent idealised. The compression borders visible on the specimen, were lost on the lithograph. Even more strikingly, the partial overlap of last order pinnae in the smaller one of the two penultimate pinnae, is not represented on the lithograph which also shows four side pinnae whereas the specimen also preserved parts of two additional pinnae of the last order, which were omitted by the artist. Although the overall representation is quite reasonable, there is an element of "the artist's impression".

The holotype of Alethopteris urophylla shows gradual lengthening of pinnules in the lower part of the larger penultimate pinna fragment. Pinnules of equivalent size and shape occur in the specimen figured here from Yorkshire, England (Fig. 5), representing the medial part of a pinna of the penultimate order. A lower position in the frond is suggested by the pinna with markedly longer pinnules and ditto terminal depicted by figure 6 (specimen from Sanquhar, Southern Uplands of Scotland). Like the holotype from Merthyr Tydfill, South Wales, it shows slightly asymmetrical pinnules with bluntly acuminate apices and narrowly confluent bases with a marked constriction on the acroscopic side. The dense, fairly regular venation is characteristic. Comparable specimens are depicted by figures $7 \mathrm{a}-\mathrm{c}$ from the Springhill Mines Formation of the Cumberland Basin in Nova Scotia, Canada. Shorter pinnules of similar characteristics to those of the holotype are shown by figure 7d, also from the Springhill Mines Formation.

Figures 8-10 represent a specimen from the North of France, figured previously by Buisine (1961: pl. XIII, fig. 1). It shows all the transitions from lengthened pinnules to small pinnae, and is more complete than the classical specimen figured by Zeiller (1886: pl. XXXI, fig. 1) from the same locality (veine Cécile).

A specimen from the Peñarroya-Belmez-Espiel Coalfield of SW Spain (Fig. 11a) also shows the longer kind of pinnules tapering into bluntly acuminate apices. The apical pinnule of this pinna fragment is broken off so that its elongate shape is not visible, but must be assumed. The well preserved veining pattern is displayed by figure $11 \mathrm{~b}$ (at $x$ 6). This allows observing generally once bifurcate lateral veins which are sometimes tripartite or in fours. Simple veins are notably absent. Another fragment, this time from NW Spain (Fig. 12), shows pinnules of similar size and shape, with a fairly regular venation. It is noted that the presence of simple veins is suggested by incomplete preservation near the midrib, where the first vein bifurcation commonly takes place.

\section{GEOGRAPHIC AND STRATIGRAPHIC DISTRIBUTION}

This chapter can only be written as a result of the analysis of the literature as reflected in the list of synonymy for Alethopteris urophylla, and the corresponding records of stratigraphic ranges in the different areas (Fig. 13). With regard to the geographic distribution, it is noted that this refers to North America and Europe, i.e. only part of the Amerosinian Realm (palaeoequatorial belt) of Pennsylvanian times. This restriction probably reflects the paucity of records corresponding to the lower Pennsylvanian in Central Asia and China with the adjacent parts of SE Asia. Although conjectural, there is no reason why the widespread Alethopteris urophylla should not occur all over the palaeoequatorial belt.

Within the area where this species has been recorded (generally under the name of Alethopteris lonchitica, auctorum, non von Schlotheim), the westernmost occurrence is in the Michigan Basin of the American Midcontinent. This is situated on the large cratonic area which extends from North America across part of northern Europe to the Russian Platform (including Scandinavia). The most extensive records correspond to the sedimentary basins which were established on and near the margin of this vast cratonic area. This was subjected, in the main, to shallow marine conditions (with cyclothemic deposits - compare Heckel, 1989, 1995, 2003). Basins accumulating predominantly non-marine, coal-bearing deposits occur mainly from the Appalachians through the Maritime Provinces of Canada into the British Isles, northern Europe (North of France, Belgium, the Netherlands, West Germany, NE Germany), Poland/Moravia, and, on the southern margin of the Russian Platform, the vast area of the Donbass (Russia/Ukraine). Although there is general agreement on the continuity between North America and Europe (the Atlantic Ocean opened up much later than the Pennsylvanian), the exact fit between the different elements of these two major areas is still subject to discussion. The various palaeotectonic elements involved are still only partly understood and the various reconstructions proposed in the literature are all debatable in detail. This is why the present authors decided against including a map showing localities.

To the South of the basinal areas linked to the cratonic margin referred to, lies the tectonically mobile region of the Hercynian (Variscan) Chain, an area of uplift with continental basins (rift? and strike-slip), which verged onto the cratonic margin. The exact extent southwards of the Hercynian Chain is also subject to discussion, but it is clear that northern Spain (Cantabrian Mountains, Pyrenees), an area on the western edge of the Palaeotethys, had a different geological history, which is not clearly linked to the Hercynian Chain. On the other hand, the various terranes constituting the Iberian Massif, did originate from areas which may have been linked to NW Eur- 
ope (Wagner, 2004). A single occurrence in the Vendée, SW France might be explained in a similar manner. More isolated occurrences, which are not clearly attributable to major palaeotectonic units, lie in the French Alps, Rumania and Bulgaria.

Whatever the palaeotectonic conditions may have been, it seems that the composition of Pennsylvanian floras is sufficiently similar throughout the North American and European area, to suggest the absence of oceanic barriers and, by inference, a continuity of land area at times of marine lowstand. Alethopteris urophylla is one of the more widespread lower Pennsylvanian floral elements.

A list of geographic locations, as justified by the list of synonymy and Figs 1-12 of the present paper, includes Michigan, Alabama, Georgia, West Virginia (Appalachians), New Brunswick, Nova Scotia (Cumberland Basin), Scotland (Midland Valley and Southern Uplands), England North of the Wales/Brabant Massif, South Wales, NW

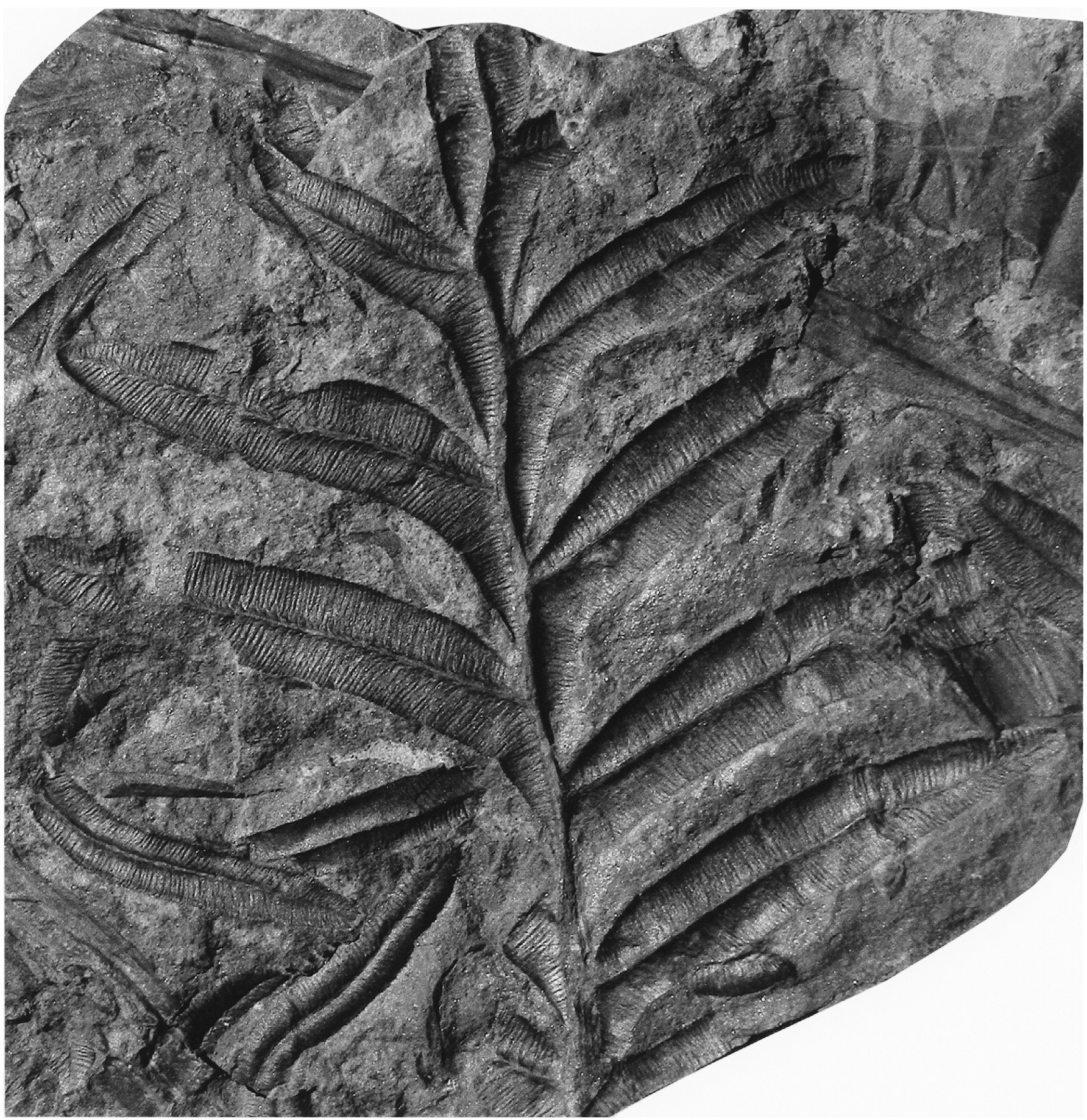

Figure 12. Near-terminal pinna fragment with elongate pinnules (x 3). Origin: Curavacas Formation at Los Cintos, south slope of Monte Hormigales, province Palencia, NW Spain, lower Duckmantian (loc. 8646). Repository: Centro Paleobotánico, Córdoba. 
France, Belgium, Netherlands Limburg, western Germany (Ruhr Coalfield), the NE Germany subsurface, La Vendée in SW France, the French Alps, NW Spain (Cantabrian Mountains), Pyrenees, SW Spain (Sierra Morena), the Upper Silesian Basin of Poland and Moravia (Czech Republic), Rumania, Bulgaria (Svoge Basin), and the Donbass of Ukraine and Russia.

The full stratigraphic ranges cannot always be given for each individual area, since in some cases only isolated records exist. The different nature of the existing records is reflected in the chart of figure 13 .

The westernmost occurrence is in the Michigan Basin, situated in the northern Midcontinent of the United States. Arnold (1949) figured and described the Pennsylvanian flora of the Michigan Basin, which covers a range from upper Langsettian to upper Duckmantian, according to his records, and using present-day stratigraphic terminology. He recorded Alethopteris urophylla as Alethopteris helenae Lesquereux, 1879. This is undoubted the wrong identification. The locality was identified as the Big Charles, probably the "Intermediate Flora" of Arnold (1949: Table I). This might be early Duckmantian.

One of the specimens figured as Alethopteris lonchitica from Alabama by Gillespie \& Rheams (1985: pl. II, fig. 2) may be attributed to Alethopteris urophylla. No exact locality is given, but the total range represented in the Warrior Basin of Alabama is stated to correspond to the New River Formation of the Appalachians (Blake et al., 2002: Fig. 5), of Langsettian age (op. cit.). A single fragment figured from Georgia by Gillespie et al. (1989) as Alethopteris lonchitica may also be attributed to Alethopteris urophylla. The coal-bearing strata in Georgia are regarded as equivalent to part of the New River, i.e. Langsettian (op. cit.).

The more complete records from West Virginia (Blake et al., 2002: Fig. 2) show Alethopteris urophylla to range from the higher part of the New River Formation to the upper Kanawha, which is regarded as equivalent to upper Langsettian, through Duckmantian into lower Bolsovian.

Alethopteris urophylla has been recorded from New Brunswick, Canada, as Alethopteris discrepans and Alethopteris lonchitica. The locality is "Fern Ledges" at Saint John, which is of Langsettian age (Wagner, 2005). Undoubted records from Nova Scotia, Canada, all refer to the Springhill Mines which worked coal seams of late Langsettian age (according to floral data in the authors' possession).

The northeastern continuation of Nova Scotia and Newfoundland lies in Scotland where Alethopteris urophylla has been documented (as Alethopteris lonchitica) from the Central Coalfield in the Midland Valley (Kidston, 1916) and from Sanquhar in the Southern Uplands (see Fig. 6 of the present paper). Kidston's record refers to Lanarkian, a term which corresponds with upper Yeadonian and lower Langsettian, according to Crookall (1955: Table A). The specimens from Sanquhar are from lower Duckmantian deposits (K. Higgs, pers. comm., 13-11-2007).

More extensive records exist from England and South Wales, both as Alethopteris lonchitica (auctorum) and Alethopteris urophylla. The stratigraphic range of Alethopteris urophylla from England North of the Wales-Brabant Massif is from Langsettian to lower Bolsovian according to Cleal (2005). Lower levels, corresponding to the Namurian substages, Marsdenian and Yeadonian, have yielded

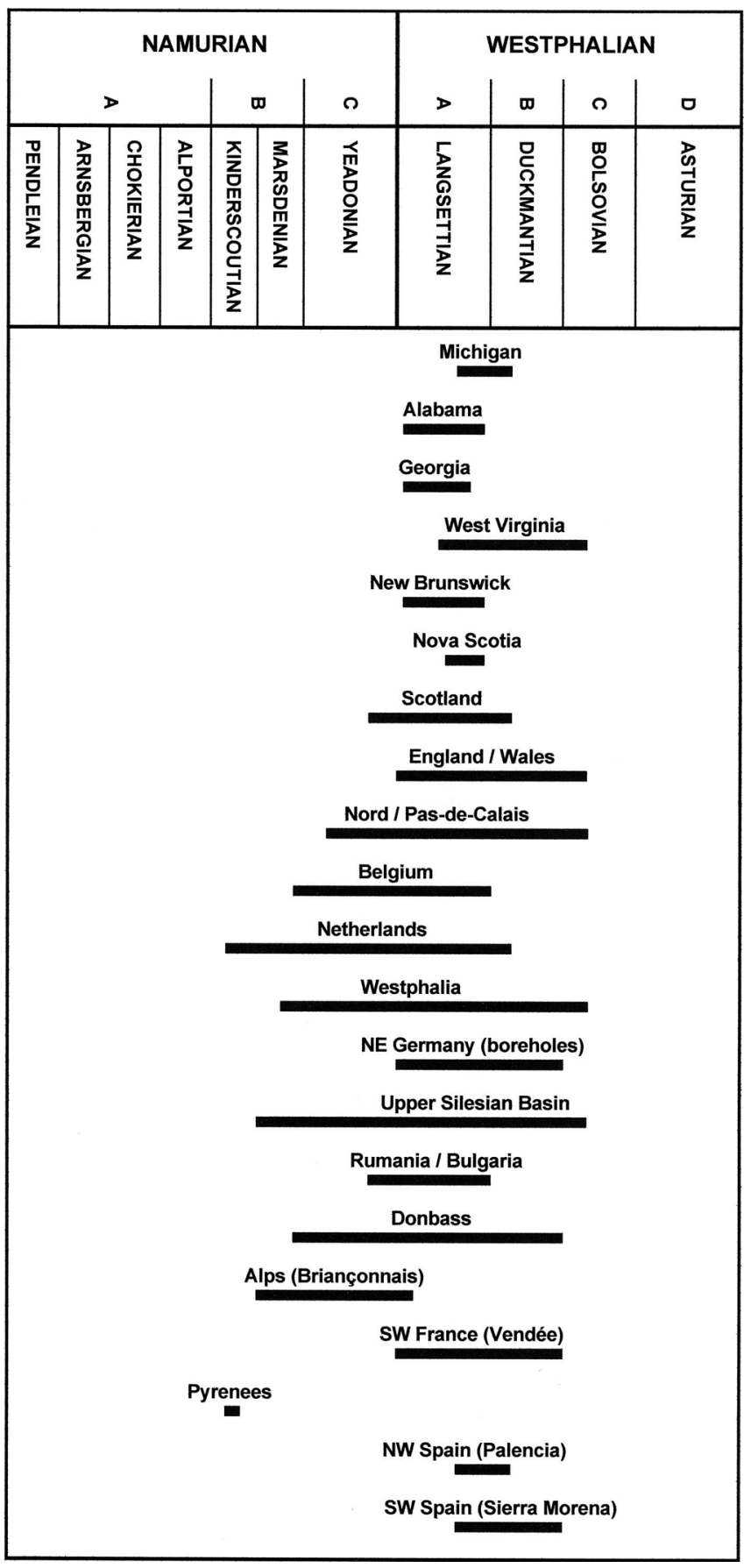

Figure 13. Chart depicting stratigraphic ranges established for the different areas in the palaeoquatorial belt where records of Alethopteris urophylla exist. 
Alethopteris lonchitica (auctorum), as recorded by Lacey (1952). In the absence of figured specimens, it is difficult to judge these records.

South of the Wales-Brabant Massif there are detailed records from the South Wales Coalfield (Cleal, 2007), showing Alethopteris urophylla to range from basal Langsettian to about mid-Bolsovian. Arber (1914) and Crookall (1955) mention Alethopteris lonchitica (auctorum) from the Kent Coalfield in England, which is the lateral continuation of the Nord/Pas-de-Calais Coalfield in northwestern France. The records of Alethopteris urophylla from Nord/ Pas-de-Calais are mainly under the name of Alethopteris lonchitica (Buisine, 1961), but it seems that some remains identified as Alethopteris serlii also belong to Alethopteris urophylla (compare list of synonymy). The ranges given by Buisine (op. cit.) show Alethopteris lonchitica (auctorum) as mainly occurring in Langsettian and lowermost Duckmantian, with a total range from Yeadonian to upper Duckmantian. The specimen figured by Buisine (1961: pl. VIII, figs 2, 2a) as Alethopteris serlii, but attributed by the present authors to Alethopteris urophylla, originated from the Faisceau d'Ernestine, Assise de Bruay, lower Bolsovian.

In Belgium, which continues the area of coal-measures in northwestern France, Alethopteris lonchitica (auctorum) has been recorded from Marsdenian (lower part of Assise d'Andenne) to Langsettian.

North of the Liège Coalfield area in Belgium lies the South Limburg Coalfield of the Netherlands and the contiguous Aachen Coalfield of western Germany. Fossil records are mainly from South Limburg and boreholes from the deeper subsurface to the North. The lowest occurrence is in the Kinderscoutian near Aachen (de Voogd, 1928). The records from South Limburg include material from Langsettian and lower Duckmantian strata (Gothan \& Jongmans in Jongmans, 1915; Jongmans, 1928).

In the Ruhr District and Westphalia (western Germany) there are records of Alethopteris lonchitica (auctorum) ranging from upper Marsdenian to lower Asturian. The lower part of the range refers to the Namurian B of HagenVorhalle in Westphalia (Schultka, 1995; Josten \& van Amerom, 2003). All illustrations of this species by Josten refer to material from Langsettian and Duckmantian strata of the Ruhr District. The higher part of the range as given by Josten (2005) refers to the lower Asturian (ex Westphalian D) of the Osnabrück region, as quoted in Josten (1966: 614) from Piesberg. The relevant specimens were not illustrated and since it may be assumed that Josten accepted the large amount of morphological variation admitted by Gothan (1953), it seems likely that forms comparable to Alethopteris westphalensis are involved. This is why the present writers do not accept a range beyond lower Bolsovian for western Germany.

Very deep boreholes in NE Germany reached coalbearing strata of Langsettian and Duckmantian ages (Da- ber, 1992). No exact stratigraphic identification is given for the specimen figured as Alethopteris sp. by Daber (1969), which the present authors include with Alethopteris urophylla.

Alethopteris lonchitica (auctorum) has been recorded repeatedly from the Upper Silesian Basin which extends from southern Poland into the Czech Republic (Moravia). In a range chart from Poland (Kotasowa \& Migier, 1995) it is stated that Alethopteris lonchitica (auctorum) occurs from upper Yeadonian to basal Bolsovian. Purkyňová (1996) indicates that the range of Alethopteris lonchitica (auctorum) in the Ostrava-Karviná Coalfield (Czech part of the Upper Silesian Basin) extends from high Namurian B (Marsdenian) to Westphalian A (Langsettian).

Two coalfield areas in the Balkans (Rumania, Bulgaria) contain records of Alethopteris urophylla. The Rumanian material came from Baia Nouă (Sirinia Basin), which was regarded as Westphalian D by Biţoianu (1972a, 1973). However, the presence of several species of Neuralethopteris which are misidentified by Biţoianu (e.g. Neuropteris ovata Hoffmann, 1826 - Biţoianu, 1973: pl. II, fig. 16, which is most likely referable to Neuralethopteris jongmansii Laveine, 1967), show that the Langsettian is involved. In Bulgaria, the Svoge Basin, North of Sofia (Tenčov, 1977), Alethopteris lonchitica (auctorum) was recorded from the Svidanya Formation of Yeadonian or Langsettian age.

Even further East, on the southern edge of the Russian Platform, lies the large basin of the Donbass. The range of Alethopteris lonchitica (auctorum) is given by Fissunenko in Solovieva et al. (1996) as embracing most of the Bashkirian (from $\mathrm{E}_{8}$ limestone) and reaching into middle Moscovian ( $\mathrm{m}_{2}$ coal bed). The correlation with West European stratigraphic units is controversial, but it is apparent that this species does not occur in upper Moscovian which would correspond approximately to Bolsovian-Asturian.

Several records of Alethopteris lonchitica (auctorum) have been published from the French Alps (Briançonnais). These refer to strata assigned to Marsdenian to lower Langsettian (Brousmiche-Delcambre et al., 1995-98).

In SW Europe there are first of all the old records from the Vendée (Bureau, 1913-14). This refers to the lower Westphalian coalfield of Chantonnay, a narrow NW-SE oriented, elongate basin alongside a major (strike-slip?) fault (see map in Mathieu, 1932).

Similar conditions obtain for two lower Westphalian coalfields in the SW Spain, viz. Villanueva del Río y Minas (Sevilla province) and Peñarroya-Belmez-Espiel (Córdoba province). Both strike NW-SE and the Peñarroya Basin is clearly controlled by a very major strike-slip fault (intracontinental, transcurrent fault? - see Wagner, 1999). The Villanueva Coalfield contains a small succession (less than a hundred metres) with floral contents indicating a late Langsettian age (see the records in García-Loygorri 
\& Ortuño Aznar, 1969, and Ortuño, 1970, who suggested early Duckmantian). The abundant floral records from the Peñarroya-Belmez-Espiel Coalfield (Álvarez-Vázquez, 1995, 2000) show Alethopteris urophylla to occur in upper Langsettian to upper Duckmantian (and lower Bolsovian?) strata.

In the Cantabrian Mountains of NW Spain the lower Westphalian floras are rarely represented due to the predominant marine facies. The sparse records are from uppermost Langsettian and lower Duckmantian of the Curavacas Conglomerate Formation in northern Palencia (Stockmans $\&$ Willière, 1965, and Fig. 12 of the present paper).

The Pyrenees provide only rare floral records of the Namurian-Westphalian interval, due to the predominant marine facies and large gaps in the stratigraphic succession. An important record is that published by Delvolvé \& Laveine (1985) from the axial zone (Laruns-Somport map sheet of the French Geological Survey). Goniatite finds from the same horizon have allowed dating their records of Alethopteris lonchitica (auctorum) as Kinderscoutian $\left(\mathrm{R}_{1}\right)$.

It follows that Alethopteris urophylla ranges from middle Namurian (Kinderscoutian) to lower Bolsovian, with most records referring to Langsettian and Duckmantian strata. This seems to reflect the predominant occurrence of coal-bearing strata in the lower Westphalian, and may not be a true reflection of the principal occurrence of this species.

\section{SUMMARY AND CONCLUSIONS}

The common lower Pennsylvanian species Alethopteris urophylla has been shown to be widespread in the coalbearing facies of the palaeoequatorial belt (Amerosinian Realm). Examples are figured from the British Isles (South Wales, Yorkshire, southern Scotland), Nova Scotia, North of France, NW and SW Spain. The photographic illustration of the holotype shows that Brongniart's illustrator took certain liberties with the drawing as published in 1834 .

Alethopteris urophylla was usually recorded as Alethopteris lonchitica, a species which Zodrow \& Cleal (1998) refigured on the basis of its lectotype from the upper Westphalian of Saarland. Unfortunately, their description is based on material from the Sydney Basin in Nova Scotia, which may have been misidentified. Indeed, they identified Alethopteris lonchitica with the Alethopteris lonchitifolia-missouriensis-westphalensis complex, but this seems to have been a mistake. The present writers suggest that the type Alethopteris lonchitica identifies with Alethopteris friedelii, which has also been described from SaarLorraine. This species has been placed in synonymy with Alethopteris ambigua by Wagner (1968). The taxonomic revision by Zodrow \& Cleal thus needs to be modified. Alethopteris lonchitica is here regarded as synonymous with Alethopteris ambigua (= Alethopteris friedelii), and different to the Alethopteris lonchitifolia-missouriensiswestphalensis complex. Alethopteris lesquereuxii needs to be reinstated and not placed in synonymy with Alethopteris ambigua (= Alethopteris lonchitica), as Zodrow \& Cleal suggest, albeit tentatively.

The geographic distribution of this species is shown to range from Michigan in the West to the Donbass in the East.

\section{ACKNOWLEDGEMENTS}

P. Shepherd and M. Howe, curators, Palaeontology Unit, British Geological Survey, at Keyworth, are thanked for the loan of the Alethopteris urophylla holotype, as is Jean Dougherty, of the Geological Survey of Canada, Ottawa, for the loan of the Canadian specimens figured here. We are grateful to Ken Higgs, Cork, Ireland, for providing specimens and locality details from the Sanquhar Coalfield (Scotland). Thanks are due to Chris Cleal for information regarding the Hutton Collection in Newcastle (England), as well as for refereeing this paper. His valuable comments as well as those of an anonymous referee have allowed focusing more sharply the main thrust of this paper, i.e. a detailed description and discussion of Alethopteris urophylla. A visit to the classical Cassville locality, West Virginia, organised by Bill Gillespie, is fondly remembered. A photograph of the holotype, produced by Barry Pigott, University of Sheffield, many years ago, is also acknowledged with pleasure. Jean-Pierre Laveine kindly provided the photographs of the specimen from the North of France, which are reproduced here. The authors are grateful for these photographs made by P. De Bleeckere at the Musée d'Histoire Naturelle de Lille. There are also grateful for drawing attention to specimens figured as Alethopteris cf. lonchitica from China. Manfred Barthel is warmly thanked for re-examining the lectotype of Alethopteris lonchitica, at the present writers' request.

\section{REFERENCES}

Álvarez-Vázquez, C. 1995. Macroflora del Westfaliense inferior de la cuenca de Peñarroya-Belmez-Espiel (Córdoba). Doctoral Thesis, Departamento de Geología, Universidad de Oviedo, I (Texto): 1-393, text-figs 1-21; II (Láminas): Láms 1-100; III (Anexos): Anexo 1: 1-7; Anexo 2: 1-111; Anexo 3: Cuadros 1-9; Anexo 4: Planos 1-4 (Unpublished).

Álvarez-Vázquez, C. 2000. Nuevos datos aportados por la macroflora a la datación y conocimiento de la cuenca carbonífera de Peñarroya-Belmez-Espiel (Córdoba, SO España). Trabajos de Geología, 21 (Volumen Homenaje a J. Truyols), 37-46.

Amerom,H.W.J. van 1975. De steenkoolflora van de koollagen GB 11 en 12 van de steenstort en in de ondergrondse ontsluiting van de mijnonderneming Laura en Vereeniging bij Eygelshoven. Grondboor en Hamer, 2, 65-76, pls 1-3.

Amerom, H.W.J. van \& Lambrecht, L. 1975. Geologische und Paläobotanisch-Palökologische Untersuchungen im West- 
fal A und B von Lüttich (Belgien). Huitième Congrès International de Stratigraphie et de Géologie du Carbonifère, Moscow 1975, Compte Rendu 3, 148-158, pls I-VIII.

Anisimova, O.I. 1979. Flora and Phytostratigraphy of the Middle Carboniferous of North Caucasus. Akademia Nauk, Ukrainskoy SSR. Institut Geologicheskikh, Naukova Dumka, Kiev, 1-108, Tab. I-XLVII (in Russian).

Arber, E.A.N. 1904. The Fossil Flora of the Culm Measures of North-west Devon, and the Palaeo-botanical Evidence with Regard to the Age of the Beds. Philosophical Transactions of the Royal Society of London, Series B, 197, 291-325, pls 19-20.

Arber, E.A.N. 1914. On the Fossil Flora of the Kent Coalfield. Quarterly Journal of the Geological Society, London, LXX, 54-81, pls XI-XIII.

Arnold, Ch.A. 1949. Fossil flora of the Michigan Coal Basin. Contributions from the Museum of Paleontology, University of Michigan, VII (9), 131-269, pls I-XXXIV.

Artis, E.T. 1825. Antediluvian Phytology, as illustrated by a Collection of the Fossil Remains of Plants peculiar to the Coal Formation of Great Britain ( $2^{\text {nd }}$ edition 1838). London, xiii + 1-24, pls I-XXIV (non vide).

Bailey, L.W. 1865. Observations on the Geology of Southern New Brunswick, made principally during the summer of 1864 by Prof. L.W. Bailey, Messrs. Geo. F. Matthew, and C.F. Hartt. Printed by order of House of Assembly, Fredericton, $185 \mathrm{pp}$. (non vide).

Barnard, P.D.W. \& Long, A.G. 1975. Triradioxylon - a New Genus of Lower Carboniferous Petrified Stems and Petioles together with a Review of the Classification of Early Pterophytina. Transactions Royal Society of Edinburgh, 69 (10), 231-249.

Barthel, M. 1962. Epidermisuntersuchungen an einigen inkohlten Pteridospermenblättern des Oberkarbons und Perms. Geologie, 11 (33), 1-140, Tafn I-XLI.

Bell, W.A. 1938. Fossil Flora of Sydney Coalfield, Nova Scotia. Geological Survey of Canada, Memoir 215, 1-334, pls I-CVII.

Bell, W.A. 1944. Carboniferous rocks and fossil floras of northern Nova Scotia. Canada Department of Mines and Resources, Mines and Geology Branch, Geological Survey, Memoir, 238, 1-119, pls I-LXXIX.

Bell, W.A. 1962. Flora of Pennsylvanian Pictou Group of New Brunswick. Geological Survey of Canada, Bulletin, 87, 1-71, pls I-LVI.

Bell, W.A. 1966. Illustrations of Canadian Fossils. Carboniferous plants of eastern Canada. Geological Survey of Canada, Paper, 66-11, 1-76, pls I-XXXVI.

Bertrand, P. 1932. Bassin houiller de la Sarre et de la Lorraine. I. Flore fossile. 2. Aléthoptéridées. Études Gîtes Minéraux de la France, 61-107, pls XXXI-LX.

Biţoianu, C. 1972a. Observaţii asupra conţinutului paleobotanic al depozitelor carbonifere din zona Baia Nouă (Banat). Studii Cercetări de Geologie, Geofizică, Geografie, Seria Geologie, 17 (1), 125-130, pls I-III.

Biţoianu, C. 1972b. Conţinutul floristic al depozitelor carbonifere din zona Sviniţa. Studii Cercetări de Geologie, Geofizică, Geografie, Seria Geologie, 17 (2), 391-399, pls I-IV.
Biţoianu, C. 1973. La flore du Carbonifère supérieur de la Roumanie. Septième Congrès International de Stratigraphie et de Géologie du Carbonifère, Krefeld 1971, Compte Rendu II, 115-127, pls I-III.

Blake, B.M. 1997. Revised Lithostratigraphy and Megafloral Biostratigraphy of the New River and Kanawha Formations (Pottsville Group: Lower and Middle Pennsylvanian) in Southern West Virginia. Master Thesis, West Virginia University, 1-159, pls 1-11 (Unpublished).

Blake, B.M., Cross, A.T., Eble, C.F., Gillespie, W.H. \& Pfefferkorn, H.W. 2002. Selected plant megafossils from the Carboniferous of the Appalachian region, eastern United States: geographic and stratigraphic distribution. In: Carboniferous and Permian of the World, Proceedings XIV International Congress Carboniferous and Permian Stratigraphy, Calgary, Alberta 1999 (Eds. L.V. Hills, C.M. Henderson \& E.W. Bamber). Canadian Society of Petroleum Geologists, Memoir, 19, 259-335, pls IXXXV.

Bode, H. 1941. Einige Bemerkungen zur Stratigraphie des Saarbrücker Karbons. Zeitschrift der Deutschen Geologischen Gesellschaft, 93 (1), 24-33.

Boulay, N. 1876. Le terrain houiller du Nord de la France et ses végétaux fossiles. Thèse de Géologie, Faculté des Sciences de Caen, 1-74, pls I-IV.

Bouroz, A. 1956. Alethopteris Bertrandi nov. sp. du Westphalien C du Pas-de-Calais. Annales de la Société Géologique du Nord, LXXV, 137-143, pls VII-IX.

Brongniart, A. 1828a. Prodrome d'une Histoire des Végétaux fossiles. F.G. Levrault, Paris, 1-225.

Brongniart, A. 1828b-1838. Histoire des végétaux fossiles, ou Recherches botaniques et géologiques sur les végétaux renfermés dans les diverses couches du globe. Texte: I (1828-1837) - 1 (1828): i-xii, 1-80; 2 (1828): 81-136; 3 (1829): 137-168; 4 (1830): 169-208; 5 (1831): 209-248; 6 (1831): 249-264; 7 (1833): 265-288; 8 (1834): 289-312; 9 (1834): 313-316; 10 (1836): 337-368; 11 (1837): 369-416; 12 (1837): 417-488. II (1837-1838) - 13 (1837): 1-24; 14 (1838): 25-56; 15 (1838): 57-72; Atlas: I - Pls 1-166; II Pls 1-30. Facsimile Edition W. Junk, Berlin (1915).

Brousmiche-Delcambre, Cl., Mercier, D. \& Coquel, R. 1995. Implications stratigraphiques de la révision de la flore carbonifère au Sud de Briançon. Compte rendus de l'Académie des Sciences, Paris, série IIa, 320, 335-340.

Brousmiche Delcambre, Cl., Mercier, D. \& Coquel, R. 1996. Révision de la flore carbonifère du Briançonnais. Implications stratigraphiques. Ière partie: Vallée de la Durance au Sud de Briançon. Palaeontographica, Abt. B, 239 (4-6), 77-107, pls 1-10.

Brousmiche Delcambre, Cl., Coquel, R. \& Mercier, D. 1997. Sur l'âge des terrains carbonifères affleurant au col de Tramouillon (Massif de Gaulent, Sud Briançonnais). Revue de Paléobiologie, 16 (1), 169-179, pls I-II.

Brousmiche Delcambre, Cl., Mercier, D. \& Coquel, R. 1998. Révision de la flore carbonifère du Briançonnais. Implications stratigraphiques. Partie II: Le Nord de Briançon, de la vallée de la Guisane à celle de la Clarée. Palaeontographica, Abt. B, 248 (4-6), 87-125, pls 1-14, text-fig. 1. 
Brousmiche Delcambre, Cl., Coquel, R., Mercier, D. \& Sartori, M. 1998. Révision du Carbonifère de Taninges (Préalpes du Chablais) - Paléobotanique - Palynologie. Revue de Paléobiologie, 17 (2), 549-563, pls I-III.

Buisine, M. 1961. Contribution à l'étude de la flore du Terrain houiller. Les Aléthoptéridées du Nord de la France. Houillères du Bassin du Nord et du Pas-de-Calais. Études géologiques pour l'Atlas de Topographie Souterraine publiées par le Service Géologique des H.B.N.P.C.I. Flore fossile, 4, 1-317, pls I-LXXIV.

Bureau, M.E. 1913-1914. Bassin de la Basse Loire. Fascicule II: Description des flores fossiles. Études des Gîtes Minéraux de la France. Texte (1914): 1-417. Atlas (1913): pls I-LXXX.

Caride, C., Greber, C. \& Ortuño, G. 1973. Flore des faisceaux productifs du bassin central des Asturies (Espagne). Septième Congrès International de Stratigraphie et de Géologie du Carbonifère, Krefeld 1971, Compte Rendu II, 267285, pls I-IV.

Cleal, C.J. 1993. Plants. 44. Gymnospermophyta. In: The Fossil Record 2 (Ed. M.J. Benton). The Palaeontological Association, Chapman \& Hall, London, 795-808.

Cleal, C.J. 2005. The Westphalian macrofloral record from the cratonic central Pennines Basin, UK. Zeitschrift der deutschen Gesellschaft für Geowissenschaften, 156 (3), 387-401.

Cleal, C.J. 2007. The Westphalian-Stephanian macrofloral record from the South Wales Coalfield, UK. Geological Magazine, 144 (3), 465-486.

Cleal, C.J. \& Shute, C.H. 1995. A synopsis of neuropteroid foliage from the Carboniferous and Lower Permian of Europe. Bulletin of The Natural History Museum, Geology Series, 51 (1), 1-52.

Cleal, C.J. \& Thomas, B.A. 1994. Plant Fossils of the British Coal Measures. Palaeontological Association, Field Guides to Fossils, 6, 1-222.

Cleal, C.J. \& Thomas, B.A. 2004. Late Carboniferous palaeobotany of the upper Bideford Formation, north Devon: a coastal setting for a Coal Measures flora. Proceedings of the Geologists' Association, 115, 267-281.

Cleal, C.J., Lazarus, M. \& Townsend, A. 2005. Illustrations and illustrators during the "Golden Age" of palaeobotany: 18001840. In: History of Palaeobotany: Selected Essays (Eds. A.J. Bowden, C.V. Burek, C.V.\& R. Wilding). Geological Society, London, Special Publications, 241, 41-61.

Coquel, R. \& Laveine, J.P. 1979. Alethopteris jankii nov. sp., du Westphalien C-D du Nord de la France. Annales Société Geólogique du Nord, XCVIII, 123-126, pls XI-XIV.

Corsin, P. 1932. Guide paléontologique dans le Terrain houiller du Nord de la France. Travaux et Mémoires de l'Université de Lille, Albums 5, 1-44, pls A-C, I-XL.

Cremer, L. 1893. Über die Fossilen Farne des Westfälischen Carbons und ihre Bedeutung für eine Gliederung des letzteren. Mitteilungen aus dem geologischen Museum der Westfälischen Berggewerkschaftskasse, 1-49, Tafn I-III.

Crookall, R. 1929. Coal Measure Plants. Edward Arnold \& Co., London, 1-80, pls I-XXXIX.

Crookall, R. 1955. Fossil Plants of the Carboniferous Rocks of Great Britain (Second Section). Memoirs Geological Survey of Great Britain. Palaeontology, IV (1), 1-84, pls I-XXIV.

Daber, R. 1969. Paläobotanische Hinweise auf eine paralisch beeinflußte Oberkarbon-Senke im tieferen Untergrund Nordostdeutschlands, II (Teil 1). Geologie, 18 (3), 253297, Tafn I-XIV.

Daber, R. 1992. Paläobotanische Hinweise auf eine paralisch beeinflußte Oberkarbon-Senke im tieferen Untergrund Nordostdeutschlands, II (Teil 2). Wissenschaftliche Zeitschrift der Humboldt-Universität zu Berlin, Mathematik/Naturwissenschaften, 41, 29-38.

Dawson, J.W. 1862. On the Flora of the Devonian Period in North-Eastern America. Quarterly Journal Geological Society of London, XVIII, 296-330, pls XII-XVII.

Dawson, J.W. 1863. Further Observations on the Devonian Plants of Maine, Gaspé, and New York. Quarterly Journal Geological Society of London, XIX, 458-469, pls XVII-XIX.

Dawson, J.W. 1868. Acadian Geology. The Geological Structure, Organic Remains, and Mineral Resources of Nova Scotia, New Brunswick, and Prince Edward Island ( $2^{\text {nd }}$ edition), MacMillan and Co., London, 1-694.

Dawson, J.W. 1871. The Fossil Plants of the Devonian and Upper Silurian Formations. Geological Survey Canada, Report, 1-92, pls I-XX.

Delvolvé, J.-J. \& Laveine, J.-P. 1985. Sur quelques flores du Carbonifère des Pyrénées béarnaises. Geobios, 18 (3), 281304, pl. A, pls 1-7.

Drägert, K. 1964. Pflanzensoziologische Untersuchungen in den Mittleren Essener Schichten des nördlichen Ruhrgebietes. Forschungsberichte des Landes Nordrhein-Westfalen, 1363, 1-295, Tafn 1-12.

Ettingshausen, C. von 1854. Die Steinkohlenflora von Radnitz in Böhmen. Abhandlugen der k. k. geologischen Reichsanstalt, II, 3 (3), 1-74, Tafn 1-29.

Fiebig, H.E.R. \& Leggewie, W. 1974. Die Namurflora des Ruhrgebietes und ihre stratigraphische Bedeutung. Septième Congrès International de Stratigraphie et de Géologie du Carbonifère, Krefeld 1971, Compte Rendu III, 45-61, Tafn 1-4.

Fontaine, W.M. \& White, I.C. 1880. The Permian or Upper Carboniferous Flora of West Virginia and S.W. Pennsylvania. Second Geological Survey of Pennsylvania, Report of Progress, 1-143, pls I-XXXVIII.

Franke, F. 1912. Beiträge zur Kenntnis der paläozoischen Arten von Alethopteris und Callipteridium. Inaugural Dissertation Berlin, 1-121, 46 figs.

Franke, F. 1913a. Alethopteris lonchitica. In: Abbildungen und Beschreibungen fossiler Pflanzen-Reste (Ed. H. Potonié). Königlichen Preußischen Geologischen Landesanstalt, Lieferung IX, 161.

Franke, F. 1913b. Alethopteris Serli. In: Abbildungen und Beschreibungen fossiler Pflanzen-Reste (Ed. H. Potonié). Königlichen Preußischen Geologischen Landesanstalt, Lieferung IX, 162.

Frech, F. 1880. Lethaea Geognostica. Handbuch der Erdgeschichte mit Abbildungen der für die Formationen bezeich- 
nendsten Versteinerungen. Herausgegeben von Geologen unter Redaktion von Fritz Frech. Schweitzerbartsche Verlag, Stuttgart, Textband I, Lieferung 1, 1-324.

García-Loygorri, A. \& Ortuño Aznar, G. 1969. Estudio geológico de la cuenca carbonífera de Villanueva del Río y Minas (Sevilla). III Jornadas y Primeras Internacionales Minero-Metalúrgicas, Gijón 1967, I, 821-864.

Gil Fernández, J. 2003. La flora de la cuenca carbonífera de Villanueva del Río y Minas (Sevilla). Subsuelo, 19, 37-42.

Gillespie, W.H. \& Pfefferkorn, H.W. 1986. The pteridosperm Alethopteris virginiana F. \& W. in the United States is the same as A. leonensis Wagner in the Stephanian of Europe. Proceedings West Virginia Academy of Science. Abstracts of Papers for the Sixty-First Annual Session, 58 (1), 25.

Gillespie, W.H. \& Rheams, L.J. 1985. Plant megafossils from the Carboniferous of Alabama, U.S.A. Dixième Congrès International de Stratigraphie et de Géologie du Carbonifère, Madrid 1983, Compte Rendu 2, 191-202, pls I-III.

Gillespie, W.H., Clendening, J.A. \& Pfefferkorn, H.W. 1978. Plant Fossils of West Virginia and Adjacent Areas. West Virginia Geological and Economic Survey, Educational Series ED-3A, 1-172, pls 1-64.

Gillespie, W.H., Crawford, T.J. \& Waters, J.A. 1989. Plant fossils of the Pennsylvanian System of Georgia. Guidebook, $38^{\text {th }}$ Annual Meeting of Southeastern Section. The Geological Society of America, Atlanta, Georgia, April 3-5, 1989, 1-13, pls 1-2.

Gómez-Alba, J.A.S. 1988. Guía de Campo de los Fósiles de España y de Europa. Editorial Omega, 925 pp. (incl. láms 1-368, láms I-XX).

Göppert, H.R. von 1836. Die Fossilen Farnkräuter. Verhandlungen der Kaiserlichen Leopoldinisch Carolinischen Akademie der Naturforscher, XXII Supl., 1-299, Tab. IXLIV.

Göppert, H.R. von 1841-1848. Die Gattungen der fossilen Pflanzen verglichen mit denen der Jetztwelt und durch Abbildungen erläutert. Bonn, Lief. I-II (1841): 1-120, Tafn IXVIII; Lief. III-IV (1842): Tafn I-XVIII; Lief. V-VI (1848): Tafn I-XX (non vide).

Gothan, W. 1909. Lonchopteris. In: Abbildungen und Beschreibungen fossiler Pflanzen-Reste (Ed. H. Potonié). Königlich Preußischen Geologischen Landesanstalt, Lieferung IX, 1-7.

Gothan, W. 1913. Die Oberschlesische Steinkohlenflora. I. Farne und farnähnliche Gewächse (Cycadofilices bezw. Pteridospermen). Abhandlungen Königlichen Preussischen Geologischen Landesanstalt, (N.F.), 75, 278 pp., Tafn 1-53.

Gothan, W. 1953. Die Steinkohlenflora der westlichen paralischen Steinkohlenreviere Deutschlands. 5. Geologisches Jahrbuch, Beiheft, 10, 1-83, Tafn 1-44.

Gothan, W. \& Franke, F. 1929. Wissenschaftliche Heimatbücher für den Westfälisch-Rheinischen Industriebezirk. Band II. Der Westfälisch-Rheinische Steinkohlenwald und seine Kohlen. Verlag Friedrich Wilhelm Ruhfus, Dortmund, 1-141, Tafn 1-52.

Gothan, W. \& Remy, W. 1957. Steinkohlenpflanzen. Leitfaden zum Bestimmen der wichtigsten pflanzlichen Fos- silien des Paläozoikums im rheinisch-westfälischen Steinkohlengebiet. Verlag Glückauf GmbH, Essen, 1-248, Tafn 1-6, Abb. 1-221.

Goubet, P., Pfefferkorn, H.W. \& Gillespie, W.H. 2000. Neuralethopterids (Trigonocarpalean Pteridosperms) from the Early Pennsylvanian of eastern North America. PaleoBios, 20 (3), 11-37.

Havlena, V. 1970. Important macroflora of Upper Namurian A and Namurian B beds between coal seams nos. 40-34 of the Ostrava-Karviná district. Věstník Ústředního ústavu geologického, 45, 95-97, pls I-II.

Havlena, V. 1984. Alethopterids of the middle and upper Namurian, Ostrava-Karviná coalfield. Časopis pro mineralogii a geologii, 29 (4), 369-377, pls I-IV.

Heckel, P.H. 1989. Updated Middle-Upper Pennsylvanian eustatic sea level curve for Midcontinent North America and preliminary biostratigraphic characterization. Onzième Congrès International de Stratigraphie et de Géologie du Carbonifère, Beijing 1987, Compte Rendu 4, 160-185.

Heckel, P.H. 1995. Glacial-eustatic base-level - Climatic model for late Middle to Late Pennsylvanian coal-bed formation in the Appalachian Basin. Journal of Sedimentary Research, $\mathbf{B 6 5}$ (3), 348-356.

Heckel, P.H. 2003. Advances in interpretive sedimentology and stratigraphy associated with study of Pennsylvanian glacial-eustatic syclothems. In: Proceedings XVth International Congress on Carboniferous and Permian Stratigraphy, Utrecht, the Netherlands, 10-16 August 2003 (Ed. Th. E. Wong). Royal Netherlands Academy of Arts and Sciences, 23-34.

Hoffmann, F. 1826. Untersuchungen über die Pflanzen-Reste des Kohlengebirges von Ibbenbühren und von Piesberge bei Osnabrück. Archiv für Bergbau und Hüttenwesen, Breslau, 13, 266-282.

Hofmann,A. \& Ryba, F. 1899. Leitpflanzen der palaeozoischen Steinkohlenablagerungen in Mittel-Europa. Atlas mit 20 Tafeln. J.G. Calve'sche k. u. k. Hof und Universitäts-Buchhandlung, Prag.

Howse, R. 1888. A Catalogue of Fossil Plants from the Hutton Collection, presented by the Council of the Mining Institute to the Natural History Society, 1883. Natural History Transactions of Northumberland, Durham, and Newcastleupon-Tyne, $\mathbf{X}, 1-135$, pls 1-6.

Janssen, R.E. 1939. Leaves and Stems from Fossil Forests. A Handbook of the Paleobotanical Collections in the Illinois State Museum. Illinois State Museum, Popular Science Series, I, 1-190, text-figs 1-165.

Jongmans, W.J. 1915. Paläobotanisch-stratigraphische Studien im Niederländischen Carbon nebst Vergleichen mit umliegenden Gebieten. Mit Anhang: Bemerkungen über einige der in den niederländischen Bohrungen gefundenen Pflanzen (W. Gothan \& W.J. Jongmans). Königlich Preußischen Geologischen Landesanstalt, 18, 1-186, Tafn I-VI.

Jongmans, W.J. 1928. Stratigraphie van het Karboon in het algemeen en van Limburg in het bijzonder. Geologisch Bureau voor het Nederlandsche Mijngebied te Heerlen, Mededeeling, 6, 1-50, pls 1-17.

Jongmans, W.J. 1932. Hoe Zuid-Limburg er in den Karboon- 
tijd uitgezien heeft. Mijnen-Nummer V.V.V., Heerlen, 1-16, Figs 1-59.

Jongmans, W.J. 1939a. Die Kohlenbecken des Karbons und Perms im USSR und Ost-Asien. Geologische Stichting, Geologisch Bureau voor het Mijngebied te Heerlen, Jaarverslag 1934-1937, 15-192, Tafn I-XL.

Jongmans, W.J. 1939b. Beiträge zur Kenntnis der Karbonflora in den östlichen Teilen des Anatolischen Kohlenbeckens. Veröffentlichung des Institutes für Lagerstaettenforschung der Türkei, Serie B, 2, 23-40, Tafn I-XIV.

Jongmans, W.J. 1952. Note sur la flore du terrain carbonifère de Djerada (Maroc oriental). Notes et Mémoires, Service géologique, Division des Mines et de la Géologie, Direction de la production industrielle et de Mines, Maroc, 91 , 1-29, pls I-XXI.

Jongmans, W.J. 1953a. Palaeontological notes on the coalfields of the province of Gelderland in the eastern Netherlands. Mededelingen Geologische Stichting, Serie C-III-1 (2), 7-25, pls 1-9.

Jongmans, W. J. 1953b. Palaeontology of the sections in the lower Westphalian A, in borings LXVI, LXVII, LXVIII in Limburg (Voerendaal) flora. Mededelingen Geologische Stichting, Serie C-III-1 (3), 29-45, pls 1-13.

Jongmans, W.J. \& Gothan, W. 1935. Die paläobotanische Ergebnisse der Djambi-Expedition 1925. Jaarboek van het Mijnwezen in Nederlandsch-Indië, 1930, Verhandelingen, 71-201, Tafn 1-58.

Josten, K.-H. 1961. Pflanzensoziologische Beobachtungen an Steinkohlenbohrungen im Ruhrgebiet. Palaeontographica, Abt. B, 108 (1-2), 39-42, Taf. 10.

Josten, K.-H. 1966. Zur Flora des jüngeren Karbons (Westfal $\mathrm{C}$ bis Stefan) in Nordwestdeutschland und ihr Vergleich mit anderen Gebieten. Fortschritte Geologie von Rheinland und Westfalen, 13, 565-644, Tafn 1-18.

Josten, K.-H. 1983. Die fossilen Floren im Namur des Ruhrkarbons. Fortschritte Geologie von Rheinland und Westfalen, 31, 1-327, Tafn 1-56.

Josten, K.-H. 1991. Die Steinkohlen-Floren Nordwestdeutschlands. Fortschritte Geologie von Rheinland und Westfalen, 36, Textband: 1-434; Tafelband: 1-220.

Josten, K.-H.2005. Florenstratigraphie des Oberkarbons in Nordwestdeutschland. In: Stratigraphie von Deutschland. V. Das Oberkarbon (Pennsylvanium) in Deutschland(Ed.V.Wrede). Courier Forschungsinstitut Senckenberg, 254, 119-132.

Josten, K.-H. \& Amerom, H.W. J. van. 2003. Die Flora des Namur B aus Hagen-Vorhalle. Geologie und Paläontologie in Westfalen, 61, 5-303, Tafn 1-117.

Kessler, P. 1916. 4. Die Alethopteriden und Mariopteriden der Saarbrücken Schichten des Saarbeckens. Zeitschrift der Deutschen Geologischen Gesellschaft, 67 (1915), 6984, Tafn IX-XIII.

Kidston, R. 1886. Catalogue of the Palaeozoic Plants in the Department of Geology and Palaeontology, British Museum (Natural History). Harrison and Sons, London, 1-288.

Kidston, R. 1888. On Neuropteris plicata, Sternberg, and Neuropteris rectinervis, Kidston, n. sp. Transactions of the Royal Society of Edinburgh, XXXV, Part I (5), 313$315,1 \mathrm{pl}$.
Kidston, R. 1901. The Flora of the Carboniferous Period. First Paper. Proceedings Yorkshire Geological and Polytechnic Society, New Series, XIV, Part II, 189-230, pls XXV-XXXVII.

Kidston, R. 1916. The Value of Fossils. In: The Economic Geology of the Central Coalfield of Scotland (Area V). Memoirs Geological Survey Scotland, X, 1-124.

Kindelán, J.A. 1957. Nota sobre la sucesión estratigráfica del hullero de Asturias, en la zona: El Viso-Olloniego-Riosa. Notas y Comunicaciones del Instituto Geológico y Minero de España, 48 (4), 91-106.

Kotasowa, A. 1995. Stop 8. Stratigraphical collection of fossil plants from the Carboniferous sequence of the Upper Silesian Coal Basin. In: Paleontology of the Carboniferous of the Upper Silesian Coal Basin and the Lublin Coal Basin. Guide to Excursion A4 (Coord. S. DybováJachowicz, A. Fijałkowska, P. Hemza, J. Kabrtova, H. Kmiecik, S. Knafel, A. Kotasowa, I. Lipiarski, T. Migier, Ł. Musiał, E. Purkyňová, F. Řehoř, A. Siewniak-Madej, J. Soboń-Podgórska, M. Tabor, G. Takla, A. Tomaś, A. Trzepierczyńska, E. Turnau, D. Zdebska \& E. Żołdani). XIII International Congress on Carboniferous-Permian, Kraków, Poland, Polish Geological Institute, 1-58, pls IVIII.

Kotasowa, A. \& Migier, T. 1995. Macroflora. In: The Carboniferous System in Poland (Eds. A. Zdanowski \& H. Żakowa). Prace Państwowego Instytutu Geologicznego, CXLVIII, 1-215.

Kvaček, Z. \& Kvaček, J. 1992. Šternberkovo dílo Flora der Vorwelt, jeho význam a stav dokladové sbírky. [Sternberg's work Flora der Vorwelt, its significance and the state of the original collection]. Časopis národního muzea v Praze, 158 (1-4), 31-42, Tab. I-VI (in Czech with English summary).

Kvaček, J. \& Straková, M. 1997. Catalogue of fossil plants described in works of Kaspar M. Sternberg. National Museum, Prague, 1-201, pl. A, pls 1-67.

Lacey, W.S. 1952. Additions to the Millstone Grit Flora of Lancashire. Troisième Congrès International de Stratigraphie et de Géologie du Carbonifère, Heerlen 1951, Compte Rendu I, 379-383.

Langford, G. 1958. The Wilmington Coal Flora from a Pennsylvanian Deposit in Will County, Illinois. Esconi Associates, 1-360, figs 1-674.

Laveine, J.-P. 1967. Contribution à l'étude de la flore du Terrain Houiller. Les Neuroptéridées du Nord de la France. Études géologiques por l'Atlas de Topographie souterraine, Service Géologique Houillères du Bassin du Nord et du Pas-de-Calais. I.- Flore fossile, 5. Texte: 1-344, pls A-H, J-K, M-P; Atlas: pls I-LXXXIV.

Laveine, J.-P. 1986. The size of the frond in the genus Alethopteris Sternberg (Pteridospermopsida, Carboniferous). Geobios, 19 (1), 49-56, pl. 1,

Laveine, J.-P., Zhang, S., Liu, L., An, D., Zheng, Q, Cao, J. \& Lemoigne, Y. 2003. The late Palaeozoic floras of the Hotan area (Xinjiang Uygur Autonomous Region, Northwest China), and their palaeogeographical significance. Revue Paléobiologie, 22 (2), 473-559, pls I-XX. 
Leary, R.L. 1976. Inventory of Type and Figured Paleobotanical Specimens in the Illinois State Museum. Illinois State Museum, Inventory of Geological Collections, $\mathrm{n}^{\circ} \mathbf{2}$, Part 2, 1-64.

Leary, R.L. \& Pfefferkorn,H.W. 1977.An Early Pennsylvanian Flora with Megalopteris and Noeggerathiales from WestCentral Illinois. Illinois State Geological Survey, Circular $\mathbf{5 0 0}, 1-77$, pls 1-15.

Lesquereux, L. 1858. Fossil Plants of the Coal Strata of Pennsylvania. In: H.D. Rogers, The Geology of Pennsylvania, II (pt. II), 835-884, pls I-XXIII.

Lesquereux, L. 1879-1884. Description of the Coal Flora of the Carboniferous Formation in Pennsylvania and throughout the United States. Second Geological Survey of Pennsylvania. Report of Progress, Text - I (1880), 1-354; II (1880), 355-694, pls LXXXVI, LXXXVII; III (1884), 695-977, pls LXXXVIII-CXI; Atlas (1879), pls A, B, pls I-LXXXV.

Lindley, J. \& Hutton, W. 1831-1837. The Fossil Flora of Great Britain; or, Figures and Descriptions of the Vegetable Remains found in a Fossil State in this Country. James Ridgway and Sons, London, I (1831-1833) - 1831: 1-47, Pls 1-14; 1832: 48-166, Pls 15-59; 1833: 167-224, Pls 6079; II (1833-1835) - 1833: 1-56, Pls 80-99; 1834: 57-156, Pls 100-137; 1835: 157-208, Pls 138-156; III (1835-1837) 1835: 1-72, Pls 157-176; 1836: 73-122, Pls 177-194; 1837 : 123-208, Pls 195-230.

Lyons, P.C., Zeissner, C.R., Barwood, H.L. \& Adinolfi, F.G. 1985. North American and European megafloral correlations with the upper part of the Pottsville Formation of the Warrior coal field, Alabama, U.S.A. Dixième Congrès International de Stratigraphie et de Géologie du Carbonifère, Madrid 1983, Compte Rendu 2, 205-245, pls I-XIV.

Mamet, B. \& Martínez, C 1981. Late Viséan microfossils of the Las Caleras Bajas limestone (Córdoba, Spain). Revista Española de Micropaleontología, XIII (1), 105-118, pls 1-3.

Mathieu, G. 1932. Observations stratigraphiques dans le Bocage Vendéen et la Gâtine. Annales de la Société Géologique du Nord, LVII, 47-69.

Matthew, G.F. 1910. Revision of the Flora of the Little River Group No. II. Transactions Royal Society of Canada, Third Series (1909-1910), III, Section IV, 77-113 (pls I-VI).

Meyen, S.V. 1984. Basic Features of Gymnosperm Systematics and Phylogeny as Evidenced by the Fossil Record. The Botanical Review, 50 (1), 1-112.

Migier, T. 1966. Charakterystyka florystyczna osadów karbonu lubelskiego. [Floristic characteristics of the deposits of the Lublin Carboniferous]. Instytut Geologiczny, Prace, XLIV, 83-101, Tab. I-X.

Migier, T. 1972. Charakterystyka florystyczna karbonu produktywnego górnoślaskiego zagłębia węglowego [A floristic description of the productive Carboniferous of the Upper Silesian Coal Basin]. Instytut Geologiczny, Prace, LXI, 135-182, Tab. I-XXII.

Nĕmejc, F. 1936. Studies on the Alethopterids of the Permocarboniferous of Central Bohemia (with remarks on forms collected in other Bohemian coaldistricts). Vĕstník Královske České Společnosti Nauk, II, 1-18, pls I, II, figs 1-10.
Novik, E.O. 1952. Coal Measure Flora of the European Part of SSSR. Akademia Nauk SSSR, I, 1-468, Tab. I-LXXI (in Russian).

Novik, E.O. 1954. Carboniferous flora of the eastern part of the Donetz Basin. Akademia Nauk, Ukrainskoi SSR. Trudi Instituta Geologicheskikh Nauk, Serie Stratigrafii i Paleontologii, vipusk 7, 1-128, Tab. I-XXVI (in Russian).

Oleksyshyn, J. 1982. Fossil plants from the Anthracite Coal Fields of Eastern Pennsylvania. Pennsylvania Geological Survey, General Geology Report, 72, 1-157.

Opluštil, S., Pšenička, J. Libertín, M. \& Šimůnek, Z. 2007. Vegetation patterns of Westphalian and Lower Stephanian mire assemblages preserved in tuff beds of the continental basins of Czech Republic. Review of Palaeobotany and Palynology, 143 (3-4), 107-154, pls I-VIII.

Ortuño, M.G. 1970. Middle Westphalian strata in south-west Spain. Sixième Congrès International de Stratigraphie et de Géologie du Carbonifère, Sheffield 1967, Compte Rendu III, 1276-1293, pls 1-2.

Potonié, H. 1893. Die Flora des Rothliegenden von Thüringen. Königlich Preussischen geologischen Landesanstalt (N.F.), 9, 1-298, Tab. I-II, Tafn I-XXXIV.

Purkyňová, E. 1957. Příspěvek k poznání květeny doubravského pásma [A contribution to the knowledge of the fossil plants of the Doubrava coal seams (Coal district of Ostrava - Karviná)]. Časopis pro mineralogii a geologii, 2 (3), 293-305.

Purkyňová, E. 1962. Flóra produktivního karbonu ostravskokarvinského revíru. Pracovní metody geologické služby, 3, 1-116, Tab. I-XXV.

Purkyňová, E. 1971. První nálezy karvinské flóry v karbonu u Frenštátu pod Radhoštěm. Časopis Slezského Muzea Opava (A), XX, 135-149, Tab. I-VIII.

Purkyňová, E. 1977. Namurian Flora of the Moravian Part of the Upper Silesian Coal Basin. In: Symposium on Carboniferous Stratigraphy (Eds. V.M. Holub \& R.H. Wagner). Ústřední ústav geologický, Praha, 289-303, pls I-IV.

Purkyňová, E. 1986. Floristická zonálnost žacléřského souvrství v důlním poli Dolu Šverma u Žacléře (vnitrosudetská pánev). Časopis Slezského Muzea Opava (A), 35, 5764, Tab. I-VI.

Purkyňová, E. 1990. K biostratigrafii karbonu v doubravských vrstvách hornoslezské černouhelné pánve. Časopis Slezského Muzea Opava (A), 39, 213-224, Tab. I-X.

Purkyňová, E. 1996. Macrofloral zonation in the Upper Silesian Coal Basin (Czech Republic). Časopis Slezského Muzea Opava (A), 45, 59-64.

Ralli, G. 1933. Le Bassin Houiller d'Héraclée et la Flore du Culm et du Houiller Moyen. Imprimerie Zellitch Frères, Péra, Part I. Bassin Houiller d'Héraclée: 1-100; Part II. La Flore du Culm et du Houiller Moyen: 99-166, pls IXXXV.

Remy, W. \& Remy, R. 1977. Die Floren des Erdaltertums. Einführung in Morphologie, Anatomie, Geobotanik und Biostratigraphie der Pflanzen des Paläophytikums. Verlag Glückauf GMBH, Essen, 1-468.

Renier, A. 1908. Les méthodes paléontologiques pour l'étude stratigraphique du terrain houiller. Revue Universelle des 
Mines, de la Métallurgie, des Travaux Publics, des Sciences et des Arts appliqués à l'Industrie, $4^{\mathrm{e}}$ série, XXI, 1-176, figs $1-70$.

Renier, A., Cambier, R., Deltenre, H. \& Schmitz, G. 1910. Documents pour l'étude de la paléontologie du terrain houiller. Soc. An. Imprimerie, H. Vaillant-Carmanne, Liège, 1-26, pls I-118.

Renier, A., Stockmans, F., Demanet, F. \& Straelen, V. van. 1938. Flore et Faune houillères de la Belgique. Introduction à l'étude paléontologique du terrain houiller. Musée royal d'Histoire naturelle de Belgique, Bruxelles. Texte: 1-317; Planches: 1-49, pls 1-144.

Roehl, D. von 1868. Fossile Flora der Steinkohlen-Formation Westphalens einschliesslich Piesberg bei Osnabrueck. $\mathrm{Pa}$ laeontographica, XVIII, 1-191, Tafn I-XXXII.

Sauveur, M. 1848. Végétaux fossiles des Terrains houillers de la Belgique. Académie Royale des Sciences, des Lettres et des Beaux-Arts de Belgique, pls I-XXXVI.

Scheihing, M.H. \& Pfefferkorn,H.W. 1980. Morphologic variation in Alethopteris (Pteridosperms, Carboniferous) from St. Clair, Pennsylvania, USA. Palaeontographica, Abt. B, 172 (1-4), 1-9, pl. 1.

Schlotheim, E.F. von 1804. Beschreibung merkwürdiger Kräuter-Abdrücke und Pflanzen-Versteinerungen (Ein Beitrag zur Flora der Vorwelt). Gotha, 1-68, Tafn I-XIV.

Schlotheim, E.F. von 1820. Die Petrefactenkunde auf ihrem jetztigen Standpunkte, durch die Beschreibung seiner Sammlung versteinerter und fossiler Überreste des Thierund Pflanzenreichs der Vorwelt erläutert. Gotha, i-lxii (Einleitung), 1-437, Tafn I-XXXVI (Nachträge in 1822; i-xi, 100 p. und 1823,114 p.).

Schultka, S. 1995. Die Flora des Namur B in der ehemaligen Ziegeleitongrube Voßacker bei Fröndenberg. Eine Übersicht. Geologie und Paläontologie in Westfalen, 35, 1-97, Tafn 1-31.

Scott, A.C. 1977. A review of the ecology of upper Carboniferous plant assemblages, with new data from Strathclyde. Palaeontology, 20 (2), 447-473, pls 50, 51.

Scott, A.C. \& Smith, D.G. 1977. What use are fossil plants? Hermathena, CXXIII, 50-67, pls 1, 2.

Scott, D.H. 1923. Studies in Fossil Botany. Vol. II. Spermophyta.A. \& C. Black Ltd., London (Third Edition), 1-446, text-figs 1-136.

Seward, A.C. 1898-1919. Fossil Plants. Cambridge Biological Series, Cambridge University Press, London, I (1898): 1-452, figs 1-111; II (1910): 1-624, figs 112-376; III (1917): 1-656, figs 377-629; IV (1919): 1-543, figs 630-818.

Šimůnek, Z. 1996. Carboniferous alethopterids of the Karviná and Žacléř Formations (Czech Republic). Sborník geologických věd, Paleontologie, 33, 5-37, pls I-XXIV, text-figs 1-41.

Šimůnek, Z. 2007. Cuticular analysis of medullosan pteridosperms from the Radnice Member (Pennsylvanian) of the Central and Western Bohemian basins (Czech Republic). In: Proceedings XVth International Congress on Carboniferous and Permian Stratigraphy, Utrecht, the Netherlands, 10-16 August 2003 (Ed. Th. E. Wong). Royal Netherlands Academy of Arts and Sciences, 389-402, pls 1-5.
Solovieva, M.N., Gubareva, V.S., Ivanova, E.A., Fissunenko, O.P., Shcherbakov, O.A. \& Einor, O.L. 1996. Moscovian Stage. In: The Carboniferous of the World. III. The former USSR, Mongolia, Middle Eastern Platform, Afghanistan, \& Iran (Eds. R.H. Wagner, C.F. Winkler Prins \& L.F. Gra-

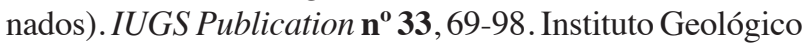
y Minero de España, Madrid.

Sternberg, K. von 1820-1838. Versuch einer geognostisch-botanischer Darstellung der Flora der Vorwelt. I (1820-1825) 1 (1820): 1-24, Tafn I-XIII; 2 (1823): 1-33, Tafn XIV-XXVI; 3 (1824): 1-40, Tafn XXVII-XXXIX; 4 (1825): 1-48, Tafn XL-LIX, Tafn A-E. II (1833-1838) - 5/6 (1833): 1-80, Tafn I-XXVI; 7/8 (1838, Pres1): 81-220, Tafn I-LXVIII, Tafn A, B (incl. Corda: Skizzen zur vergleichenden Phytotomie vorund jetztweltlicher Pflanzenstämme, I-LXXI).

Stockmans, F. \& Willière, Y. 1951. Quelques végétaux namuriens et westphaliens du Charbonnage d'Aiseau-Presle. Association pour l'Étude de la Paléontologie et de la Stratigraphie houillères, 9, pls A-D.

Stockmans, F. \& Willière, Y. 1952-1953. Végétaux namuriens de la Belgique. Association pour l'Étude de la Paléontologie et de la Stratigraphie houillères, 13. Texte (1953), 1-382. Atlas (1952), 1-12, pls I-LVII.

Stockmans, F. \& Willière, Y. 1958. Le niveau dit à Sphenopteris hoeninghausi du Bassin Houiller de Liège. In: Étude géologique du Bassin Houiller de Liège. La Concession Belle-Vue et Bienvenue (A. Lhoest). Association pour l'Étude de la Paléontologie et de la Stratigraphie houillères, 36, pls I-V.

Stockmans, F. \& Willière, Y. 1961. Végétaux du Westphalien A de la Belgique. Graines, inflorescences et synanges. Centre national de Géologie houillère, Bruxelles, $\mathbf{4}$, 1-118, pls I-XI.

Stockmans, F. \& Willière, Y. 1965. Documents paléobotaniques pour l'étude du houiller dans le Nord-Ouest de l'Espagne. [Documentos paleobotánicos para el estudio de la geología hullera del Noroeste de España]. Mémoires, Institut Royal des Sciences Naturelles de Belgique, Deuxième Série, fasc. 79, 1-92, pls I-XXXVIII.

Stopa, S.Z. 1957. Les feuilles de fougères (Pteridophylla) du Namurien supérieur et du Westphalien le plus bas dans le bassin houiller de la Haute Silésie. Instytut Geologiczny, Prace, XIII, 1-208, Tab. I-XXXVII (in Polish with French summary).

Stopes, M.C. 1914. The "Fern Ledges" Carboniferous Flora of St. John, New Brunswick. Geological Survey of Canada, Memoir 41 (38 of Geological Series), 11-42, pls I-XXV. (Traduction française 1917. Flore carbonifère des "assises à fougères" de Saint-Jean, Nouveau-Brunswick. Geological Survey of Canada, Memoir 41).

Stur, D. 1877. Beiträge zur Kenntniss der Flora der Vorwelt. Die Culm-Flora der Ostrauer und Waldenburger Schichten. Abhandlungen der kaiserlich-königlichen geologischen Reichsanstalt, VIII (2), 1-366, Tafn I-XXVII.

Šusta, V. 1927. Phytopaläontologische Neuheiten aus dem Ostrauer-Karviner Karbon. Sborník Přirodovědecké společnosti Moravské Ostravě, IV, 1-5, Tafn I-II.

Šusta, V. 1928. Stratigraphie des Ostrau-Karviner Steinkoh- 
lenreviers im Lichte der Paläontologie. In: Der Kohlenbergbau des Ostrau-Karviner Steinkohlenreviers (Direcktoren-Konferenz des Ostrau-Karviner Steinkohlenreviers in Mährisch Ostrau im Zehnten Jahre der Čechoslovakischen Republik), 381-484, Tafn I-LXXIV.

Tenčov, Y. 1977. Flora und Biostratigraphie des Oberkarbons im Svoge-Becken (VR Bulgarien). Schriftenreihe für Geologische Wissenschaften, Heft 7, 1-163, Tafn I-XXIII.

Thomas, B.A. \& Cleal, C.J. 1993a. Middle Westphalian plant fossils from the West Cumberland Coalfield, UK. Geological Journal, 28, 101-123.

Thomas, B.A. \& Cleal, C.J. 1993b. The Coal Measures Forests. National Museum of Wales, South Western Printers, $1-32$.

Vinassa de Regny, P. \& Gortani, M. 1905. Fossili carboniferi del M. Pizzul e del Piano di Lanza nelle Alpi Carniche. Bollettino della Società geologica d'Italia, XXIV, 461520, Tav. XII-XV.

Voogd, N. de 1928. Gliederung und Fossilführung des tieferen Oberkarbons in der Umgebung von Aachen und den angrenzenden Gebieten von Holland und Belgien. Jaarverslag 1928, Geologisch Bureau voor het Nederlandsche Mijngebied, Heerlen, 11-72, Tafn I-V.

Wagner, R.H. 1959. Pecopteris pseudobucklandi ANDRAE and its generical affinities. Mededelingen Geologische Stichting, (N.S.), 12 (for 1958), 25-30, pls 14-16.

Wagner, R.H. 1961. Some Alethopterideae from the South Limburg Coalfield. Mededelingen Geologische Stichting, (N.S.), 14, 5-13, pls 1-8.

Wagner, R.H. 1964. Stephanian floras in NW. Spain, with special reference to the Westphalian D - Stephanian A boundary. Cinquième Congrès International de Stratigraphie et de Géologie du Carbonifère, Paris 1963, Compte Rendu II, 835-851, pls I-III.

Wagner, R.H. 1968. Upper Westphalian and Stephanian Species of Alethopteris from Europe, Asia Minor and North America. Mededelingen Rijks Geologische Dienst, (C), III-1-6, 1-188, pls 1-64.

Wagner, R.H. 1983. The palaeogeographical and age relationships of the Portuguese Carboniferous floras with those of other parts of the western Iberian Peninsula. In: The Carboniferous of Portugal (Eds. M.J. Lemos de Sousa \& J.T. Oliveira). Memórias dos Serviços Geológicos de Portugal, 29, 153-177, text-fig. 1, tab. 1-14.

Wagner, R.H. 1999. Peñarroya, a strike-slip controlled basin of early Westphalian age in Southwest Spain. Věstník Českého geologického ústavu, 74 (2), 3-23.

Wagner, R.H. 2001. Fósiles vegetales. Serie Recursos Naturales, $\mathbf{n}^{\circ}$ 6. Departamento de Medio Ambiente y Protección Civil, Diputación de Córdoba, 1-105.
Wagner, R.H. 2004. The Iberian Massif: a Carboniferous assembly. Journal of Iberian Geology, 30, 93-108.

Wagner, R.H. 2005. Alethopteris lancifolia Wagner, a rare element of the lower Westphalian "Fern Ledges" of Atlantic Canada. Revista Española de Paleontología, 20 (1), 15-19.

Wagner, R.H. \& Bowman, M.B.J. 1983. The position of the Bashkirian/Moscovian boundary in West European chronostratigraphy. Newsletters on Stratigraphy, 12 (3), 132-161.

Wagner, R.H. \& Mayoral, E.J. 2007. The Early Permian of Valdeviar in Sevilla province, SW Spain: basin history and climatic/palaeogeographic implications. Journal of Iberian Geology, 33 (1), 93-124.

Wagner, R.H., Carballeira, J., Ambrose, T. \& Martínez García, E. 1984. Mapa Geológico de España E. 1:50.000, Hoja $n^{o}$ 107 (Barruelo de Santullán). Memoria explicativa. Instituto Geológico y Minero de España, Madrid, 1-113

Weiss, Ch.E. 1869-1872. Fossile Flora der jüngsten Steinkohlenformation und des Rothliegenden im Saar-Rhein-Gebiete. Verlag von A. Henry, Bonn, Text (1869): 1-250. Atlas (1869-72): Tafn I-XX.

White, D. 1899. Fossil Flora of the Lower Coal Measures of Missouri. United States Geological Survey, Monographs, XXXVII, 1-467, pls I-LXXIII.

Zalessky, M.D. 1907a. Contributions à la flore fossile du terrain houiller du Donetz. I. Plantes fossiles de la collection de V. Domherr. Bulletins du Comité Géologique, XXVI, 351-422, Tab. XXII-XVII (in Russian with comments in French).

Zalessky, M.D. 1907b. Contributions à la flore fossile du terrain houiller du Donetz. II. Plantes fossiles de l'Institut géologique de l'Université Impériale de Kharkow et du Musée du Don à Novotcherkask. Bulletins du Comité Géologique, XXVI, 423-494, Tab. XVIII-XXIII (in Russian with comments in French).

Zalessky, M.D. \& Tchirkova, H.Th. 1938. The fossil flora of the middle section of the Carboniferous rocks of the Donetz Basin. Transactions of the Central Geological and Prospecting Institute, 98, 1-169, Tab. I-VII (in Russian with English summary).

Zeiller, R. 1886-1888. Bassin houiller de Valenciennes. Description de la flore fossile. Études des Gîtes Minéraux de la France. Texte (1888): 1-729. Atlas (1886): pls I-XCIV.

Zodrow, E.L. \& Cleal, C.J. 1998. Revision of the Pteridosperm foliage Alethopteris and Lonchopteridium (Upper Carboniferous), Sydney Coalfield, Nova Scotia, Canada. Palaeontographica, Abt. B, 247, 65-122, pls 1-14.

Manuscrito recibido: 3 de Marzo, 2008 Manuscrito aceptado: 24 de Julio, 2008 